\title{
Existence of Stiffness Matrix Integrals for Singularly Parameterized Domains in Isogeometric Analysis
}

\author{
T. Takacs ${ }^{\mathrm{a}, *}$, B. Jüttler ${ }^{\mathrm{b}}$ \\ a Doctoral Program “Computational Mathematics”, Johannes Kepler University, Linz, Austria \\ ${ }^{b}$ Institute of Applied Geometry, Johannes Kepler University, Faculty of Natural Sciences and Engineering, Altenberger Strasse 69, 4040 Linz, Austria
}

\begin{abstract}
Isogeometric Analysis is a numerical simulation method which uses the NURBS based representation of CAD models. NURBS stands for non-uniform rational B-splines and is a generalization of the concept of B-splines. The isogeometric method uses the tensor product structure of 2- or 3-dimensional NURBS functions to parameterize domains, which are structurally equivalent to a rectangle or a hexahedron. The special case of singularly parameterized NURBS surfaces and NURBS volumes is used to represent non-quadrangular or non-hexahedral domains without splitting, which leads to a very compact and convenient representation.

If the parameterization of the physical domain is available, the test functions for the Isogeometric Analysis are obtained by composing the inverse of the domain parameterization with the NURBS basis functions. In the case of singular parameterizations, however, some of the resulting test functions are not well defined at the singular points and they do not necessarily satisfy the required integrability assumptions. Consequently, the stiffness matrix integrals which occur in the numerical discretizations may not exist.

After summarizing the basics of the isogeometric method, we discuss the existence of the stiffness matrix integrals for 1-, 2- and 3-dimensional second order elliptic partial differential equations. We consider several types of singularities of NURBS parameterizations and derive conditions which guarantee the existence of the required integrals. In addition, we present cases with diverging integrals and show how to modify the test functions in these situations.
\end{abstract}

Keywords: singular parameterization, existence, stiffness matrix integral, isogeometric analysis, NURBS, CAD

\section{Introduction}

In various engineering applications, two major tasks are the modeling and the numerical simulation of processes and phenomena which are described by partial differential equations acting on non-trivial geometric objects. In many cases, the shape of a technical object or domain of interest is modeled via tools from Computer Aided Design (CAD). On the other hand, a widely used framework to simulate physical or technical processes, such as fluid flow or deformation of an object, is the Finite Element Method (FEM). In the classical finite element approach a mesh, containing geometric primitives such as triangles, tetrahedra or hexahedra, has to be generated from the CAD model. If the boundary of the physical domain is partly curved, then the mesh generation creates only an approximation of the domain. Also, mesh generation for real-world CAD objects can be computationally expensive and may require extensive human interaction for model repair. Therefore, a numerical simulation framework that can eliminate the need for this task is of great interest. Isogeometric Analysis, which was introduced by Hughes et al. [1], has the potential to address these problems and has become an active field of research in the area of numerical simulation.

\footnotetext{
*Corresponding author, Tel.: +43 732/2468-4086

Email addresses: thomas.takacs@jku. at (T. Takacs), bert.juettler@jku.at (B. Jüttler)
}

In Computer Aided Design the considered objects are usually represented by non-uniform rational B-splines (NURBS). The isogeometric method applies numerical methods directly to this representation of CAD models, since the parameterization and the function spaces for the numerical simulation are built up by the same basis functions. Various applications of the isogeometric method have been studied, including problems in fluid dynamics [2,3], in particular concerning the simulation of blood flow [4], simulations in computational electromagnetics [5], modeling deformation or vibration of solid structures [68] and the application to shape optimization [9-11]. One may especially consider problems where a high degree of smoothness is required, like [12], where the isogeometric method has numerous advantages compared to higher order Finite Element Methods.

Recent developments include the introduction of the concept of T-splines for Isogeometric Analysis [13, 14], which forms the basis for methods extending the classical adaptive hrefinement to the isogeometric method, see $[15,16]$. In another line of research, fundamental contributions concerning consistency and stability of the method have been provided [17-20].

In this work we focus on the applicability of the numerical methods in the case of singular parameterizations. In many situations one cannot avoid singularly parameterized objects or domains. This is due to the tensor-product structure of higherdimensional NURBS functions, which cannot implement non- 
quadrangular or non-hexahedral domains directly. Several studies on the influence of mesh distortions or on the model quality have been performed (e.g. [19, 21, 22]). The presence of singularities in the parameterization can be caused by intrinsic properties of the geometry of the object or by distortions of regular parameterizations, possibly due to mesh adaption in shape optimization. In this case, singularities can be avoided via some constraints. On the other hand, if a single-patch parameterization is used to represent non-quadrangular or non-hexahedral domains (e.g., circular domains or spheres), then singularities are necessarily present [23-25].

In this work we study whether, in the case of singular parameterizations, the basis functions from Isogeometric Analysis are suitable for the numerical simulation. We analyze sufficient conditions which guarantee that the basis functions belong to the required function spaces. We start with preliminaries concerning the considered problems and the theoretical background in Section 2. Auxiliary results and notations are presented in Section 3. We specify configurations of the control points that lead to $H^{1}$-basis functions for special cases mentioned in Section 4 and in Section 5, for the one- and twodimensional case, respectively. In Section 6 we compare different approaches to parameterize special geometric objects. Summing up, we develop a general framework to prove the existence of the stiffness matrix integrals from the numerical simulation.

\section{Preliminaries}

We shortly introduce the model problem, which is a second order elliptic partial differential equation (PDE). We consider the partial differential equation on a bounded and open domain. On this domain we define a function space in which we can solve the differential equation properly. This function space can then be discretized, using the isogeometric method. In Isogeometric Analysis the same basis functions are used for the parameterization of the domain and for the discretized function space. Concerning the formulation of the method and the considered problems we follow previous papers, especially $[1,16,22]$. The structure of this paper and the basic ideas of the presented proofs follow and extend [26].

\subsection{Numerical background}

For $d \in \mathbb{Z}^{+}$let $\Omega \subseteq \mathbb{R}^{d}$ be an open and bounded domain with Lipschitz boundary $\partial \Omega=\Gamma=\Gamma_{D} \cup \Gamma_{N}$. We consider a second order elliptic partial differential operator $L$ and a linear operator $l$, describing the boundary conditions. The boundary operator defines Dirichlet boundary conditions on $\Gamma_{D}$ and Neumann boundary conditions on $\Gamma_{N}$. Further on let $f: \Omega \rightarrow \mathbb{R}$ and $g: \Gamma \rightarrow \mathbb{R}$ be sufficiently smooth functions. For this setting we analyze the following differential equation.

Model Problem 2.1 Find $u: \bar{\Omega} \rightarrow \mathbb{R}$ such that

$$
\begin{array}{rlrl}
L u(\xi) & =f(\xi) & & \forall \xi \in \Omega \\
l u(\xi) & =g(\xi) & \forall \xi \in \Gamma .
\end{array}
$$

Similarly to the classical FEM (see [27]), we can derive a variational (weak) formulation from the general PDE. To construct the variational formulation, the differential equation (1) is multiplied with specific test functions and integrated over the domain $\Omega$. The boundary condition (2) is incorporated properly. Let $V(\Omega)=H^{1}(\Omega)$ be the underlying Hilbert space for both the space of test functions and the solution space, i.e.

$$
\begin{aligned}
& V_{0}(\Omega)=\left\{v \in V(\Omega):\left.l v\right|_{\Gamma_{D}}=0\right\} \\
& V_{g}(\Omega)=\left\{v \in V(\Omega):\left.l v\right|_{\Gamma_{D}}=\left.g\right|_{\Gamma_{D}}\right\} .
\end{aligned}
$$

The function space $H^{1}(\Omega)$ is defined by

$$
H^{1}(\Omega)=\left\{v \in L^{2}(\Omega):|\nabla v| \in L^{2}(\Omega)\right\}
$$

where the derivatives can be interpreted in a weak sense only. With the use of the $H^{1}$-seminorm

$$
|v|_{1}=\|\nabla v\|_{L^{2}}
$$

the Hilbert space norm in $H^{1}$ is defined via

$$
\|v\|_{1}^{2}=\|v\|_{L^{2}}^{2}+|v|_{1}^{2}
$$

We derive a bilinear form $a(\cdot, \cdot): V_{g} \times V_{0} \rightarrow \mathbb{R}$ and a linear functional $\langle F, \cdot\rangle: V_{0} \rightarrow \mathbb{R}$ such that the differential equation in Problem 2.1 is equivalent (under suitable assumptions, see below) to the following variational problem.

Model Problem 2.2 (Variational formulation) Find $u \in V_{g}(\Omega)$ such that

$$
a(u, v)=\langle F, v\rangle \quad \forall v \in V_{0}(\Omega) .
$$

More precisely, a solution of the classical formulation solves the weak formulation if certain integrability conditions are fulfilled. A weak solution solves the classical formulation if certain differentiability conditions are fulfilled. We refer to [27] for a full analysis of the problem.

A typical example for a second order elliptic PDE is the following model problem.

Example 2.3 Find $u: \bar{\Omega} \rightarrow \mathbb{R}$ such that:

$$
\begin{aligned}
-\Delta u & =f & & \text { in } \Omega \\
u & =g_{D} & & \text { on } \Gamma_{D} \\
\nabla u . \mathbf{n} & =g_{N} & & \text { on } \Gamma_{N} .
\end{aligned}
$$

In this formulation, $\mathbf{n}$ is the outer normal vector on $\Gamma_{N}$ and $\Delta u$ is the Laplacian of $u$. For this setting we get

$$
a(u, v)=\int_{\Omega} \nabla u . \nabla v \mathrm{~d} \xi
$$

and

$$
\langle F, v\rangle=\int_{\Omega} f v \mathrm{~d} \xi+\int_{\Gamma_{N}} g_{N} v \mathrm{~d} s .
$$


We assume that the bilinear form $a$ and the linear functional $F$ are bounded. Since the operator $L$ (as in equation (1)) is elliptic, the bilinear form $a(\cdot, \cdot)$ is coercive, i.e.

$$
\exists \mu>0: \quad \mu\|u\|^{2} \leq a(u, u),
$$

and therefore the theorem of Lax-Milgram can be applied to Problem 2.2 to show the existence and uniqueness of the solution.

Now we provide some insight into the theoretical background of Isogeometric Analysis. The isogeometric method is a special approach to the discretization of partial differential equations on non-trivial geometries. Most numerical methods are based on Galerkin's principle, which can be interpreted in the following way. Finite-dimensional function spaces $V_{g, h} \subseteq V_{g}$ and $V_{0, h} \subseteq$ $V_{0}$ are set up to solve the discretized model problem.

Model Problem 2.4 Find $u_{h} \in V_{g, h}(\Omega)$ such that:

$$
a\left(u_{h}, v_{h}\right)=\left\langle F, v_{h}\right\rangle \quad \forall v_{h} \in V_{0, h}(\Omega) .
$$

The choice of the subspaces $V_{g, h}$ and $V_{0, h}$ (or its basis functions) is called a Galerkin discretization. Solving the discretized problem rather than the original problem is called a Galerkin method. In Isogeometric Analysis the basis functions spanning $V_{0, h}$ and $V_{g, h}$ are constructed from B-spline or NURBS functions. From now on we assume that $V_{g, h}=V_{0, h}=V_{h}$.

For a given basis $\left\{\psi_{i}\right\}_{i=0}^{n-1}$ of $V_{h} \subseteq V$ one can represent any function $u_{h} \in V_{h}$ as

$$
u_{h}=\sum_{i=0}^{n-1} \psi_{i} \hat{u}_{i} .
$$

Thus $u_{h}$ can uniquely be described by its coefficient vector $\left(\hat{u}_{0}, \ldots, \hat{u}_{n-1}\right)=\hat{\mathbf{u}} \in \mathbb{R}^{n}$. The stiffness matrix $\mathbf{A}$ is defined by

$$
\mathbf{A}=\left(a_{i, j}\right)_{i, j=0}^{n-1}, \text { with } a_{i, j}=a\left(\psi_{j}, \psi_{i}\right)
$$

and the load vector $\mathbf{F}$ by

$$
\mathbf{F}=\left(F_{i}\right)_{i=0}^{n-1}, \text { with } F_{i}=\left\langle F, \psi_{i}\right\rangle .
$$

Using this notation, one can easily see that the Model Problem 2.4 is equivalent to the linear system

$$
\mathbf{A} \hat{\mathbf{u}}=\mathbf{F} .
$$

Any numerical method fitting into the framework of Galerkin methods is defined by the choice of a basis $\left\{\psi_{i}\right\}_{i=0}^{n-1}$. The method can be applied if one can ensure the existence of the matrix $\mathbf{A}$ and the vector $\mathbf{F}$. In the case of coercive operators, as in the variational formulation Problem 2.2, this is equivalent to $\psi_{i} \in H^{1}(\Omega)$ for all $i=0, \ldots, n-1$.

\subsection{B-splines and NURBS}

B-spline- or NURBS-functions are polynomial or rational functions, respectively, defined over some parameter space $\Omega_{0}$. In Isogeometric Analysis they are used to set up the Galerkin discretization. We will adopt the notation from [16]. For a precise and detailed theoretical background on B-splines and NURBS in computer aided geometric design we refer the reader to [28-30].

First we consider B-splines, which are determined by some given degree $p \in \mathbb{N}$ and a knot vector $\Theta=\left(\theta_{0}, \ldots, \theta_{m-1}\right)$. The knot vector is a non-decreasing sequence of length $m \in \mathbb{N}$ of real numbers. For $n=m-p-1$ and $i=0, \ldots, n-1$ the $i$-th $\mathrm{B}$-spline $B_{i, p}$ of degree $p$ is defined by the recurrence

$$
\begin{aligned}
& B_{i, 0}(x)= \begin{cases}1 & \text { for } \theta_{i} \leq x \leq \theta_{i+1} \\
0 & \text { else }\end{cases} \\
& B_{i, p}(x)=\frac{x-\theta_{i}}{\theta_{i+p}-\theta_{i}} B_{i, p-1}(x)+\frac{\theta_{i+p+1}-x}{\theta_{i+p+1}-\theta_{i+1}} B_{i+1, p-1}(x),
\end{aligned}
$$

where all fractions with a zero denominator are considered to be equal to zero. The support of each B-spline $B_{i, p}$ is the interval $\left[\theta_{i}, \theta_{i+p+1}\right]$. The parameter space is set to be $\left.\Omega_{0}=\right] \theta_{p}, \theta_{m-p-1}[$, which covers the support of each B-spline, except for the boundary intervals $\left[\theta_{0}, \theta_{p}\right]$ and $\left[\theta_{m-p-1}, \theta_{m-1}\right]$.

The concept of B-splines can be generalized by introducing non-uniform rational B-splines (NURBS) of degree $p$. If weights $w_{i} \in \mathbb{R}^{+}$are given, the $i$-th non-uniform rational $\mathrm{B}$ spline $R_{i, p}$ is defined by

$$
R_{i, p}(x)=\frac{B_{i, p}(x) w_{i}}{\sum_{j=0}^{n-1} B_{j, p}(x) w_{j}} .
$$

In order to extend the concept of B-splines to two dimensions one can introduce bivariate tensor product B-splines. Consequently, a degree and a knot vector is set for each direction. We consider a degree $\mathbf{p}=\left(p_{1}, p_{2}\right)$, a knot vector $\Theta=\left(\Theta^{(1)}, \Theta^{(2)}\right)$, with $\Theta^{(1)} \in \mathbb{R}^{m_{1}}$ and $\Theta^{(2)} \in \mathbb{R}^{m_{2}}$, and set $\left(n_{1}, n_{2}\right)=\mathbf{n}=\mathbf{m}-\mathbf{p}-\mathbf{1}$. Using the notation $\mathbf{i}=(i, j)$ and $\mathbf{x}=(x, y)^{T}$, the $\mathbf{i}$-th bivariate $\mathrm{B}$-spline of degree $\mathbf{p}$ and knot vector $\boldsymbol{\Theta}$ is defined by

$$
\begin{aligned}
\mathrm{B}_{\mathbf{i}, \mathbf{p}}: \boldsymbol{\Omega}_{0} & \rightarrow \mathbb{R} \\
\mathbf{x} & \mapsto B_{i, p_{1}}(x) B_{j, p_{2}}(y),
\end{aligned}
$$

for $\mathbf{0} \leq \mathbf{i} \leq \mathbf{n}-\mathbf{1}$. Here $B_{i, p_{1}}$ is the $i$-th $\mathrm{B}$-spline corresponding to $p_{1}$ and $\Theta^{(1)}$, and $B_{j, p_{2}}$ is the $j$-th B-spline corresponding to $p_{2}$ and $\Theta^{(2)}$. The parameter space $\boldsymbol{\Omega}_{0}$ is defined by

$$
\left.\mathbf{\Omega}_{0}=\right] \theta_{p_{1}}^{(1)}, \theta_{m_{1}-p_{1}-1}^{(1)}[\times] \theta_{p_{1}}^{(2)}, \theta_{m_{2}-p_{2}-1}^{(2)}[.
$$

As in the one-dimensional case, NURBS functions $\mathbf{R}_{\mathbf{i}, \mathbf{p}}$ are defined by

$$
\mathbf{R}_{\mathbf{i}, \mathbf{p}}(\mathbf{x})=\frac{\mathrm{B}_{\mathbf{i}, \mathbf{p}}(\mathbf{x}) w_{\mathbf{i}}}{\sum_{\mathbf{j}=\mathbf{0}}^{\mathbf{n}-\mathbf{1}} \mathrm{B}_{\mathbf{j}, \mathbf{p}}(\mathbf{x}) w_{\mathbf{j}}}
$$

for some positive weights $w_{\mathbf{i}} \in \mathbb{R}^{+}$.

Remark 2.5 If all weights $w_{\mathbf{i}}$ are equal, then the NURBS function $\mathrm{R}_{\mathbf{i}, \mathbf{p}}$ simplifies to the B-spline $\mathrm{B}_{\mathbf{i}, \mathbf{p}}$. Consequently, B-spline functions are special instances of NURBS functions, independent of the dimension $d$. 


\subsection{Isogeometric analysis}

In this section we recall the definition and the notations of Isogeometric Analysis. The formal notation is presented independent from the dimension $d$ of the physical space $\Omega$, but follows the notational standards for the multivariate case. In the following the dimension is considered to be $d=1$ or $d=2$, where for $d=1$ the standards for one-dimensional variables are used (e.g. write $x$ rather than $\mathbf{x}$ ).

We set the index space $\mathbb{I}$ to

$$
\mathbb{I}=\left\{\mathbf{i} \in \mathbb{N}^{d}: \mathbf{0} \leq \mathbf{i} \leq \mathbf{n}-\mathbf{1}\right\}
$$

and the parameter space to $\left.\Omega_{0}=\right] 0,1\left[{ }^{d}\right.$ without loss of generality. The parameterization $\mathbf{G}$ of $\Omega$ defined by

$$
\begin{aligned}
\mathbf{G}: \Omega_{0} & \rightarrow \mathbb{R}^{d} \\
\mathbf{x} & \mapsto \sum_{\mathbf{i} \in \mathbb{I}} \mathbf{P}_{\mathbf{i}} \phi_{\mathbf{i}}(\mathbf{x}),
\end{aligned}
$$

is defined by basis functions $\phi_{\mathbf{i}}: \Omega_{0} \rightarrow \mathbb{R}$ and control points $\mathbf{P}_{\mathbf{i}} \in \mathbb{R}^{d}$ for each $\mathbf{i} \in \mathbb{I}$. In Isogeometric Analysis the functions $\phi_{\mathbf{i}}$ are usually NURBS of some given degree $\mathbf{p}$. The physical space $\Omega$ is represented as the image of $\Omega_{0}$ under $\mathbf{G}$, i.e. $\mathbf{G}\left(\Omega_{0}\right)=\Omega$. In case of a bijective and continuously differentiable parameterization $\mathbf{G}$ (with $C^{1}$-inverse) the test functions, i.e. the basis functions of the function space $V_{h} \subset\{v: \Omega \rightarrow \mathbb{R}\}$, are defined by

$$
\psi_{\mathbf{i}}=\phi_{\mathbf{i}} \circ \mathbf{G}^{-1}: \Omega \rightarrow \mathbb{R}
$$

Figure 1 gives an overview of the functions $\mathbf{G}, \phi_{\mathbf{i}}$ and $\psi_{\mathbf{i}}$.

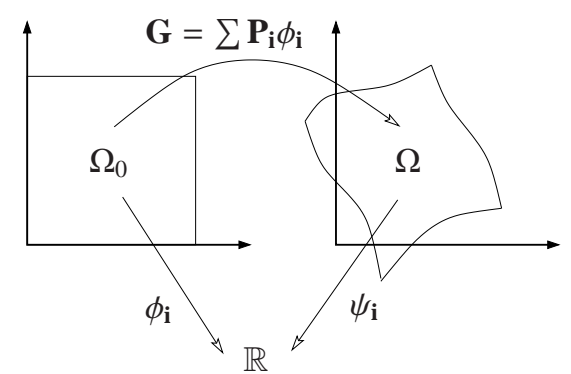

Figure 1: Scheme for the two-dimensional parameterization $\mathbf{G}$ with parameter space $\Omega_{0}$, physical domain $\Omega$ and basis functions $\phi_{\mathbf{i}}$ and $\psi_{\mathbf{i}}$

Each $v_{h} \in V_{h}$ possesses a representation in the basis $\left\{\psi_{\mathbf{i}}\right\}_{\mathbf{i} \in \mathbb{I}}$, i.e. a vector $\left(\hat{v}_{\mathbf{i}}\right)_{\mathbf{i} \in \mathbb{I}}=\hat{\mathbf{v}} \in \mathbb{R}^{\mathbf{n}}$ such that

$$
v_{h}(\xi)=\sum_{\mathbf{i} \in \mathbb{I}} \psi_{\mathbf{i}}(\xi) \hat{v}_{\mathbf{i}}
$$

for all $\xi \in \Omega$. In the one-dimensional case we will set $\phi_{i}=R_{i, p}$ and in the two-dimensional case $\phi_{\mathbf{i}}=\mathrm{R}_{\mathbf{i}, \mathbf{p}}$.

\subsection{Existence analysis}

This paper provides criteria, which guarantee that the methods from Isogeometric Analysis can be applied for a specific set of basis functions $\phi_{\mathbf{i}}$ and control points $\mathbf{P}_{\mathbf{i}}$. To guarantee the applicability of the numerical method it is necessary that the function space $V_{h}(\Omega)$ is a subspace of $H^{1}(\Omega)$. We will focus on the following problem.
Problem 2.6 For which configurations of the control points $\left(\mathbf{P}_{\mathbf{j}}\right)_{\mathbf{j} \in \mathbb{I}}$ do the test functions fulfill $\psi_{\mathbf{i}} \in H^{1}(\Omega)$ ?

Consequently, we have to verify that the test functions $\psi_{\mathbf{i}}$ are in $H^{1}(\Omega)$. More precisely, we will derive conditions on the control points that guarantee the existence of some or all of the stiffness matrix integrals. A related problem has been considered in [21], where the influence of distortions of the control point mesh on the regularity of the parameterization has been analyzed.

Clearly, in the case of NURBS the functions $\psi_{\mathbf{i}}$ are in $L^{2}(\Omega)$, as their graphs are bounded rational curves or surfaces. Hence we only have to verify if $\nabla \psi_{\mathbf{i}}$ is an $L^{2}$-function, Problem 2.6 simplifies to the following.

Problem 2.7 (Existence problem) For which configurations of the control points $\left(\mathbf{P}_{\mathbf{j}}\right)_{\mathbf{j} \in \mathbb{I}}$ do the squares of the seminorms $\left|\psi_{\mathbf{i}}\right|_{1}^{2}=$ $\left\|\nabla \psi_{\mathbf{i}}\right\|_{L^{2}}^{2}$ exist?

It is more convenient to analyze the square of the seminorm instead of the seminorm itself. Note that

$$
\left|\psi_{\mathbf{i}}\right|_{1}^{2}=\|\left.\nabla \psi_{\mathbf{i}}\right|_{L^{2}} ^{2}=\int_{\Omega} \nabla \psi_{\mathbf{i}} \cdot \nabla \psi_{\mathbf{i}} \mathrm{d} \boldsymbol{\xi}
$$

is the stiffness matrix integral $a_{\mathbf{i}, \mathbf{i}}$ for the model problem in Example 2.3. The existence of $\left\|\nabla \psi_{\mathbf{i}}\right\|_{L^{2}}^{2}$ means that the underlying $L^{2}$-norm-integral is bounded. If the parameterization G : $\Omega_{0} \rightarrow \Omega$ is regular in every point of $\bar{\Omega}_{0}$, then all test functions are in $H^{1}(\Omega)$. If singularities occur this is not true in general. Unfortunately, one cannot state general and simple conditions to answer the Existence Problem 2.7 for singular parameterizations. We will instead state and validate sufficient conditions for some classes of configurations, which are specified in the next chapters. Those model cases represent the most commonly used singular parameterizations in Isogeometric Analysis. The presented proofs can be modified and applied to larger classes of singular parameterizations.

\section{Assumptions and conditions}

In some situations, singular parameterizations cannot be avoided, for instance if one wants to parameterize a triangular domain or the interior of a circle. The latter has been done in [22], in which different ways to parameterize specific geometric objects were compared. We will state theoretical results to some of the analyzed problems in [22]. Additional conditions on the parameters and on the parameterization in general have to be satisfied in order to show basic results.

\subsection{General assumptions}

We consider open knot vectors only, i.e. the first and last knot is repeated $p+1$ times, which leads to the interpolation of the B-spline on the boundary. Without loss of generality all knot 
vectors (for $v=1,2$ ) fulfill

$$
\begin{aligned}
\theta_{0}^{(v)}=\ldots=\theta_{p_{v}}^{(v)}=0 & \\
\theta_{m_{v}-p_{v}-1}^{(v)}=\ldots= & \theta_{m_{v}-1}^{(v)}=1 \\
\theta_{j}^{(v)} \leq \theta_{j+1}^{(v)} & \text { for } 0 \leq j \leq m_{v}-1 \\
\theta_{j}^{(v)}<\theta_{j+p_{v}}^{(v)} & \text { for } 1 \leq j \leq m_{v}-2 .
\end{aligned}
$$

The parameterization $\mathbf{G}$ is continuously differentiable on $\Omega_{0}$ and its inverse is differentiable on $\Omega$. The parameterization $\mathbf{G}$ but not necessarily its inverse can be extended continuously to $\bar{\Omega}_{0}$ with $\mathbf{G}\left(\bar{\Omega}_{0}\right)=\bar{\Omega}$. We also assume that $\mathbf{G}: \bar{\Omega}_{0} \rightarrow \bar{\Omega}$ is bijective. If restricted to the interior of the domain, this condition is always required for the analysis. To fulfill all these requirements, the parameterization does not have to be regular. A parameterization $\mathbf{G}: \bar{\Omega}_{0} \rightarrow \bar{\Omega}$ is called singular in $\mathbf{x}_{0} \in \bar{\Omega}_{0}$, if

$$
\operatorname{det} \nabla \mathbf{G}\left(\mathbf{x}_{0}\right)=0
$$

Note that the values of the parameterization $\mathbf{G}$ and of the gradient $\nabla \mathbf{G}$ at boundary points are defined by their respective limits.

We assume that $\mathbf{G}$ is singular at the origin $\mathbf{x}_{0}=\mathbf{O}$ and that it is regular in $\bar{\Omega}_{0} \backslash \mathbf{x}_{0}$. Without loss of generality $\mathbf{G}\left(\mathbf{x}_{0}\right)=\mathbf{O}$. The condition "det $\nabla \mathbf{G}(\mathbf{x}) \geq 0$ " is necessary for the bijectivity of the parameterization. The bijectivity on the closed sets is not fulfilled for the class of parameterizations given in Chapter 6.1, but we can still state conditions to solve the existence problem in that case.

\subsection{Integrability condition}

In order to solve Problem 2.7, some preliminary considerations are needed. In the case of singularly parameterized domains, the integrand $\left|\nabla \psi_{\mathbf{i}}(\mathbf{x})\right|^{2}$ of the integral in equation (4) may tend to infinity for $\mathbf{x} \rightarrow \mathbf{x}_{0}$. We need to specify the behavior of the integrand around the singularity in order to prove the existence of the integral. To characterize the asymptotic behavior of a function we will use a slight modification of the classical Landau notation.

Definition 3.1 Let $f, g \in C\left(\Omega_{0}\right)$, with $g \neq 0$ in $\Omega_{0}$. Let

$$
U_{\delta}\left(\mathbf{x}_{0}\right)=\left\{\mathbf{x} \in \Omega_{0}:\left\|\mathbf{x}-\mathbf{x}_{0}\right\| \leq \delta\right\}
$$

be the $\delta$-neighborhood of $\mathbf{x}_{0}$.

- If $\forall \epsilon>0 \exists \delta>0$ :

$$
\left|\frac{f(\mathbf{x})}{g(\mathbf{x})}-1\right| \leq \epsilon \quad \forall \mathbf{x} \in U_{\delta}\left(\mathbf{x}_{0}\right)
$$

we say that the function $f$ is asymptotically equal to $g$ near $\mathbf{x}_{0}$, in symbols $f \sim g$.

- If $\exists C>0 \exists \delta>0$ :

$$
|f(\mathbf{x})| \leq C|g(\mathbf{x})| \quad \forall \mathbf{x} \in U_{\delta}\left(\mathbf{x}_{0}\right)
$$

we say that $f$ is of the order of $g$ near $\mathbf{x}_{0}$, in symbols $f \in$ $\mathrm{O}_{\mathbf{x}_{0}}(g)$.
- If $\forall \epsilon>0 \exists \delta>0$ :

$$
|f(\mathbf{x})| \leq \epsilon|g(\mathbf{x})| \quad \forall \mathbf{x} \in U_{\delta}\left(\mathbf{x}_{0}\right)
$$

we say that $g$ dominates $f$ near $\mathbf{x}_{0}$, in symbols $f \in \mathrm{o}_{\mathbf{x}_{0}}(g)$.

In the presence of only one singular point we shall write $f \in$ $\mathrm{O}(g)$ and $f \in \mathrm{O}(g)$. Note that " $\sim$ " is an equivalence relation on the function space $\tilde{C}\left(\Omega_{0}\right)=\left\{f \in C\left(\Omega_{0}\right): f \neq 0\right\}$.

The transformation of the considered integral to the parameter space $\Omega_{0}$ can always be written as an integral of the form

$$
\int_{\Omega_{0}} \frac{f(\mathbf{x})}{g(\mathbf{x})} \mathrm{d} \mathbf{x}
$$

which is the integral of a fraction, where the numerator is nonnegative and bounded from above and the denominator might tend to zero at some points.

Theorem 3.2 (Integrability condition) Let

$$
\begin{aligned}
& f, g \in C\left(\bar{\Omega}_{0}\right), \\
& \bar{f}, \bar{g} \in C\left(\Omega_{0}, \text { piecewise }\right),
\end{aligned}
$$

with $f, g, \bar{f}, \bar{g} \geq 0$. There exists a point $\mathbf{x}_{0} \in \bar{\Omega}_{0}$, where

$$
g\left(\mathbf{x}_{0}\right)=\bar{g}\left(\mathbf{x}_{0}\right)=0 .
$$

In all other points we have $f, g, \bar{f}, \bar{g}>0$. Assume that

- $f \in \mathrm{O}_{\mathbf{x}_{0}}(\bar{f})$ and

- $g \sim \bar{g}$

for the singular point $\mathbf{x}_{0}$. Under these assumptions, $f$ and $g$ fulfill

$$
\int_{\Omega_{0}} \frac{f(\mathbf{x})}{g(\mathbf{x})} \mathrm{d} \mathbf{x}<\infty,
$$

provided that $\bar{f}$ and $\bar{g}$ fulfill

$$
\int_{\Omega_{0}} \frac{\bar{f}(\mathbf{x})}{\bar{g}(\mathbf{x})} \mathrm{d} \mathbf{x}<\infty .
$$

Proof. A sketch of the proof can be found in the Appendix.

This theorem states that the existence of an integral of a rational function depends only on the asymptotic behavior of the numerator and denominator around the singularity. The result from the theorem can be generalized to cases which contain finitely many singular points.

\subsection{Assumptions on the asymptotic behavior}

A description of the asymptotic behavior of the basis functions $\phi_{\mathbf{i}}(\mathbf{x})$ near the singularity $\mathbf{x}_{0}$ is necessary for the analysis. To describe the asymptotic behavior we examine the oneand the two-dimensional case separately. In the case of a onedimensional domain we need the following definition. 
Definition 3.3 (NAM family, 1D) A set of univariate functions $\left(\phi_{i}(x)\right)_{i \in \mathbb{I}}$ is called a normalized asymptotically monomial family (NAM family) of degree $p$ near $x_{0}$ if there exist positive constants $a_{i}$ for all $0 \leq i \leq p$ such that

$$
\phi_{i}(x) \sim a_{i}\left(x-x_{0}\right)^{i}
$$

and

$$
\sum_{i \in \mathbb{I}} \phi_{i}(x)=1
$$

in a neighborhood of $x_{0}$.

If $\left(\phi_{i}\right)_{i \in \mathbb{I}}$ is a NAM family of degree $p$ near $x_{0}=0$ it follows from (6) that $\phi_{i}^{\prime} \sim i a_{i} x^{i-1}$ for $i \geq 1$. Using the condition from equation (7) we get

$$
\phi_{0}^{\prime}(x)=-\sum_{k \geq 1} \phi_{k}^{\prime}(x) \sim-\sum_{k \geq 1} c_{k} x^{k-1} \sim-c_{1}
$$

Hence there exist positive constants $c_{i}>0$ for $i=1, \ldots, p$, such that

$$
\begin{aligned}
\phi_{0}^{\prime}(x) & \sim-c_{1}, \\
\phi_{1}^{\prime}(x) & \sim c_{1}, \text { and } \\
\phi_{k}^{\prime}(x) & \sim c_{k} x^{k-1} \text { for } k=2, \ldots, p .
\end{aligned}
$$

If the corresponding knot vector fulfills equation (5) it can be shown that NURBS of degree $p$ form a NAM family of degree $p$ near $x_{0}=0$. In this case all functions $\phi_{k}$ with $k>p$ are equal to 0 near $x_{0}$.

In the two-dimensional case we require a tensor product structure to define a normalized asymptotically monomial family near the singularity $\mathbf{x}_{0}$.

Definition 3.4 (NAM family, 2D) A set of bivariate functions $\left(\phi_{\mathbf{i}}(\mathbf{x})\right)_{\mathbf{i} \in \mathbb{I}}$ is called a normalized asymptotically monomial family of degree $\mathbf{p}=\left(p_{1}, p_{2}\right)$ near $\mathbf{x}_{0}=\left(x_{0}, y_{0}\right)^{T}$ if there exist $a_{i}>0$ and $b_{j}>0$ for all $0 \leq i \leq p_{1}$ and $0 \leq j \leq p_{2}$ such that

$$
\phi_{(i, j)}(x, y) \sim a_{i} b_{j}\left(x-x_{0}\right)^{i}\left(y-y_{0}\right)^{j}
$$

and

$$
\sum_{(i, j) \in \mathbb{I}} \phi_{(i, j)}(x, y)=1
$$

in a neighborhood of $\mathbf{x}_{0}$.

Similar to the one-dimensional case we can derive results for the derivatives of the functions $\phi_{(i, j)}$. If $\left(\phi_{(i, j)}\right)_{(i, j) \in \mathbb{I}}$ is a NAM family of degree $\mathbf{p}$ near $\mathbf{x}_{0}=\mathbf{O}$ then

$$
\begin{aligned}
\nabla \phi_{(0,0)}(x, y) & \sim\left(\begin{array}{c}
-a_{1} b_{0} \\
-a_{0} b_{1}
\end{array}\right), \\
\nabla \phi_{(0, j)}(x, y) & \sim\left(\begin{array}{c}
-a_{1} b_{j} y^{j} \\
j a_{0} b_{j} y^{j-1}
\end{array}\right), \\
\nabla \phi_{(i, 0)}(x, y) & \sim\left(\begin{array}{c}
i a_{i} b_{0} x^{i-1} \\
-a_{i} b_{1} x^{i}
\end{array}\right), \text { and } \\
\nabla \phi_{(i, j)}(x, y) & \sim\left(\begin{array}{c}
i a_{i} b_{j} x^{i-1} y^{j} \\
j a_{i} b_{j} x^{i} y^{j-1}
\end{array}\right)
\end{aligned}
$$

for $1 \leq i \leq p_{1}$ and $1 \leq j \leq p_{2}$. This result can be shown similarly to the one-dimensional case by analyzing mixed derivatives of the double sum.

One can show that NURBS of degree $\mathbf{p}$ are a normalized asymptotically monomial family of degree $\mathbf{p}$ near $\mathbf{x}_{0}=\mathbf{O}$. In this case all functions $\phi_{(i, j)}$ with $i \geq p_{1}+1$ or $j \geq p_{2}+1$ fulfill $\phi_{(i, j)}=0$ in a neighborhood of $\mathbf{x}_{0}$.

\subsection{Structurally equivalent parameterizations}

We introduce a framework to show existence results for general parameterizations where the $H^{1}$-seminorm integrals can not be computed explicitly. Results can be derived if the general parameterization is structurally equivalent to a reference parameterization where existence results are known.

Definition 3.5 Two parameterizations $\hat{\mathbf{G}}$ and $\mathbf{G}$ are called structurally equivalent if $\hat{\mathbf{G}}\left(\mathbf{G}^{-1}\right)$ is regular and orientationpreserving and there exist constants $\underline{\lambda}$ and $\bar{\lambda}$ satisfying $0<\underline{\lambda}<$ $\bar{\lambda}$ such that $\mathbf{J}=\nabla\left(\hat{\mathbf{G}}\left(\mathbf{G}^{-1}\right)\right)$ fulfills $\underline{\lambda} \mathbf{I} \leq \mathbf{J}^{T} \mathbf{J} \leq \bar{\lambda} \mathbf{I}$ in $\Omega$.

In this definition the inequalities are interpreted as spectral inequalities of symmetric matrices and $\mathbf{I}$ is the identity matrix of dimension $d$. We say that under these conditions the matrix $\mathbf{J}^{T} \mathbf{J}$ is uniformly positive definite. For structurally equivalent parameterizations we can show the following.

Theorem 3.6 If two parameterizations $\hat{\mathbf{G}}$ (with test functions $\hat{\psi}_{\mathbf{i}}$ on $\hat{\Omega}$ ) and $\mathbf{G}$ (with test functions $\psi_{\mathbf{i}}$ on $\Omega$ ) with common basis functions $\phi_{\mathbf{i}}$ on $\Omega_{0}$ and common index set $\mathbb{I}$ are structurally equivalent, then $\psi_{\mathbf{i}} \in H^{1}(\Omega)$ if and only if $\hat{\psi}_{\mathbf{i}} \in H^{1}(\hat{\Omega})$.

Proof. We have

$$
\left|\psi_{\mathbf{i}}\right|_{1}^{2}=\int_{\Omega}\left\|\nabla \psi_{\mathbf{i}}(\boldsymbol{\xi})\right\|^{2} \mathrm{~d} \boldsymbol{\xi}
$$

and

$$
\begin{aligned}
\left|\hat{\psi}_{\mathbf{i}}\right|_{1}^{2} & =\int_{\hat{\Omega}}\left\|\nabla \hat{\psi}_{\mathbf{i}}(\hat{\boldsymbol{\xi}})\right\|^{2} \mathrm{~d} \hat{\boldsymbol{\xi}} \\
& =\int_{\Omega}\left\|\mathbf{J}^{-T} \nabla \psi_{\mathbf{i}}\right\|^{2} \operatorname{det}(\mathbf{J}) \mathrm{d} \boldsymbol{\xi} \\
& =\int_{\Omega}\left\langle\left(\mathbf{J}^{T} \mathbf{J}\right)^{-1} \nabla \psi_{\mathbf{i}}, \nabla \psi_{\mathbf{i}}\right\rangle \sqrt{\operatorname{det}\left(\mathbf{J}^{T} \mathbf{J}\right)} \mathrm{d} \boldsymbol{\xi}
\end{aligned}
$$

for $\mathbf{J}=\nabla\left(\hat{\mathbf{G}}\left(\mathbf{G}^{-1}\right)\right)$. Since $\mathbf{J}^{T} \mathbf{J}$ is uniformly positive definite we can bound the seminorms by

$$
\left(\underline{\lambda}^{\frac{d}{2}} / \bar{\lambda}\right)\left|\psi_{\mathbf{i}}\right|_{1}^{2} \leq\left|\hat{\psi}_{\mathbf{i}}\right|_{1}^{2} \leq\left(\bar{\lambda}^{\frac{d}{2}} / \underline{\lambda}\right)\left|\psi_{\mathbf{i}}\right|_{1}^{2}
$$

Hence $\psi_{\mathbf{i}} \in H^{1}(\Omega)$ if and only if $\hat{\psi}_{\mathbf{i}} \in H^{1}(\hat{\Omega})$.

If both parameterizations are in NURBS form, then this condition can be guaranteed by representing the determinant det $(J)$ as a NURBS function. If all coefficients and weights are positive, then the condition is satisfied.

The framework from this chapter is applied to specific examples presented in Subsections 4.3 and 5.3. 


\section{The one-dimensional case}

In this section we consider the one-dimensional differential equation (1) and answer the Existence Problem 2.7 for one specific class of singular parameterizations. The core ideas can be generalized to higher dimensions.

The given functions $\phi_{i}: \Omega_{0} \rightarrow \mathbb{R}$ for $i \in \mathbb{I}$ form a NAM family of degree $p$ near $x_{0}=0$ (such as NURBS of degree $p$ with open knot vectors). From $\phi_{i}$ we construct the test function

$$
\psi_{i}(\xi)=\phi_{i} \circ G^{-1}(\xi)
$$

We assume that the first $\alpha$ control points are equal, so a part of the control polygon degenerates to one point. This condition on the control points causes the parameterization to be singular in the point $x_{0}=0$. In fact, this is the most general case, which still fulfills all the assumptions from Subsection 3.1. In the onedimensional case a singular parameterization does not lead to a different geometry of the domain as compared to the geometry of the parameter domain, hence it is of little practical use but still of some theoretical interest.

\subsection{Existence results}

The existence of the square of the $H^{1}$-seminorm of $\psi_{i}$ has to be analyzed for each $i \in \mathbb{I}$. Under the assumption that $G$ is $C^{1}$-invertible the integral can be transformed from the physical space $\Omega=G\left(\Omega_{0}\right)$ to the parameter space $\Omega_{0}$, hence

$$
\begin{aligned}
\left|\psi_{i}\right|_{1}^{2} & =\int_{\Omega}\left(\frac{\partial \psi_{i}}{\partial \xi}\right)^{2} \mathrm{~d} \xi \\
& =\int_{\Omega_{0}}\left(\frac{\partial \phi_{i}}{\partial x}\left(\frac{\partial G}{\partial x}\right)^{-1}\right)^{2}\left(\frac{\partial G}{\partial x}\right) \mathrm{d} x \\
& =\int_{0}^{1}\left(\frac{\partial \phi_{i}}{\partial x}\right)^{2}\left(\frac{\partial G}{\partial x}\right)^{-1} \mathrm{~d} x
\end{aligned}
$$

Thus, the square of the seminorm is an integral of a fraction of two functions

$$
\left|\psi_{i}\right|_{1}^{2}=\int_{0}^{1} \frac{N_{i}(x)}{D(x)} \mathrm{d} x
$$

where

$$
N_{i}(x)=\left(\phi_{i}^{\prime}(x)\right)^{2}
$$

and

$$
D(x)=G^{\prime}(x) .
$$

The asymptotic behavior of the integrand can be computed explicitly, since the functions $\phi_{i}$ are a NAM family of degree $p$ near $x_{0}=0$ (as in Definition 3.3). The asymptotic behavior of the numerator $N_{i}$ is

$$
N_{i}(x)=\left(\phi_{i}^{\prime}(x)\right)^{2} \sim c_{i}^{2} x^{\max (2(i-1), 0)},
$$

where $c_{0}=-c_{1}$. From the definition of $G(x)$ it follows that the denominator $D$ is asymptotically equal to

$$
D(x)=G^{\prime}(x) \sim c_{0} P_{0}+\sum_{i=1}^{p} c_{i} P_{i} x^{i-1} .
$$

Existence results for basic cases can be derived using this asymptotic representation. We examine those cases where the first $\alpha$ control points are equal.

Theorem 4.1 (Existence, $1 \mathrm{D}$ case) Let $\alpha \in \mathbb{Z}^{+}$, with $2 \leq \alpha \leq p$. Assume that the control points satisfy

- $P_{i}=0$, for $0 \leq i \leq \alpha-1$, and

- $P_{\alpha} \neq 0$,

then

- $\psi_{k} \notin H^{1}(\Omega)$ for $0 \leq k \leq\left\lfloor\frac{\alpha}{2}\right\rfloor$ and

- $\psi_{k} \in H^{1}(\Omega)$ for $k>\left\lfloor\frac{\alpha}{2}\right\rfloor$.

Proof. First we analyze the behavior of the denominator. For $0 \leq i \leq \alpha-1$ the control points fulfill $P_{i}=0$, hence we can conclude from equation (10) that

$$
D(x) \sim c_{0} P_{0}+\sum_{i=0}^{p} c_{i} P_{i} x^{i-1} \sim \sum_{i=\alpha}^{p} c_{i} P_{i} x^{i-1},
$$

and with $P_{\alpha} \neq 0$ it follows

$$
D(x) \sim c_{\alpha} P_{\alpha} x^{\alpha-1} .
$$

Since the previous analysis shows that $N_{0}$ and $N_{1}$ are asymptotically equal, we do not have to treat the case $k=0$ separately, but can assume $k \geq 1$ from now on. From the integrability condition Theorem 3.2 and from equation (9) it follows that the $H^{1}$-seminorm of $\psi_{k}$ exists if and only if the integral

$$
\frac{c_{k}^{2}}{c_{\alpha} P_{\alpha}} \int_{0}^{1} x^{2(k-1)-(\alpha-1)} \mathrm{d} x
$$

exists, which is equivalent to $2 k-\alpha-1>-1$. Hence $\psi_{k}$ is in $H^{1}(\Omega)$ if and only if $k>\frac{\alpha}{2}$.

In this proof we analyzed the asymptotic behavior of the integrand around the singularity. This strategy can be generalized to other singular points $x \neq x_{0}$. In Theorem 4.1 we assume that the first $\alpha$ control points degenerate to one point, which results in basis functions that are not in $H^{1}(\Omega)$. Therefore numerical methods cannot be applied directly.

The following example illustrates these results.

Example 4.2 Consider a one-dimensional parameterization of degree $p=3$ with knot vector

$$
\Theta=\left(0,0,0,0, \frac{1}{2}, 1,1,1,1\right)
$$

and control points as in Table 1.

\begin{tabular}{rrrrrr}
\hline & $i=0$ & $i=1$ & $i=2$ & $i=3$ & $i=4$ \\
\hline$P_{i}$ & 0 & 0 & 1 & 2 & 3 \\
\hline
\end{tabular}

Table 1: Control points for Example 4.2 

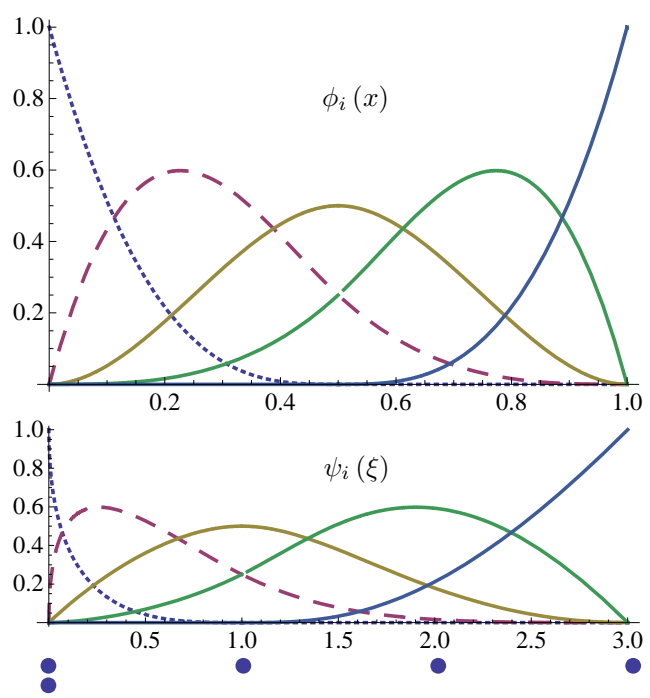

Figure 2: Functions $\phi_{i}$ on $\Omega_{0}, \psi_{i}$ on $\Omega$ and control points $P_{i}$ for $i=0, \ldots, 4$

We have $\left.\Omega_{0}=\right] 0,1[$ and $\Omega=] 0,3[$. We consider B-Splines $\phi_{i}$ on $\Omega_{0}$ and test functions $\psi_{i}$ on $\Omega$ for $i=0, \ldots, 4$, which are shown in Figure 2. The graphs of the functions $\phi_{0}$ and $\psi_{0}$ are dotted lines and the graphs of $\phi_{1}$ and $\psi_{1}$ are dashed lines.

Theorem 4.1 states that in this case $\psi_{0}$ and $\psi_{1}$ are not in $H^{1}(\Omega)$. The integrands of the stiffness matrix integrals corresponding to the test functions are shown in Figure 3. The two diverging functions are the integrands corresponding to $\psi_{0}$ (dotted line) and $\psi_{1}$ (dashed line), respectively.

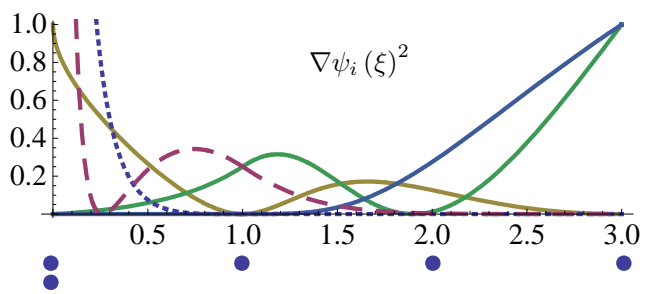

Figure 3: Integrands $\left(\nabla \psi_{i}(\xi)\right)^{2}$ on $\Omega$ for $i=0, \ldots, 4$

\subsection{Modification of the function space}

In the case of singular parameterizations it may happen that some of the test functions are not in $H^{1}$, as we have already seen. So, generally, one cannot ensure that the discretized function space $V_{h}$ is a subspace of $H^{1}$. For special cases, however, a method to cope with this problem can be developed. One possible way would be to simply omit those basis functions in $V_{h}$ that are not in $H^{1}$. In this case Dirichlet boundary conditions might not be implemented correctly. Hence, the function space $V_{h}$ has to be modified in a different way to maintain a full numerical analysis without losing to much information.

Considering the special case of Theorem 4.1, a new finitedimensional function space $\tilde{V}_{h}$, fulfilling $\tilde{V}_{h} \subseteq V_{h}$ and $\tilde{V}_{h} \subseteq H^{1}$, is set up. Therefore, we construct a new function on $\Omega_{0}$ from the sum of the old ones, setting

$$
\Phi_{A}(x)=\sum_{i=0}^{A} \phi_{i}(x)
$$

with $A=\left\lfloor\frac{\alpha}{2}\right\rfloor$. A similar approach was taken in the examples in [1]. Using this notation, the parameterization fulfills

$$
G(x)=\Phi_{A}(x) P_{0}+\sum_{i=A+1}^{n-1} \phi_{i}(x) P_{i} \quad \text { for } x \in \Omega_{0},
$$

since the first $\alpha$ control points are equal by assumption and $A<$ $\alpha$. The new basis functions on the physical domain are defined as

$$
\begin{aligned}
& \Psi_{A}(\xi)=\Phi_{A}\left(G^{-1}(\xi)\right) \\
& \Psi_{i}(\xi)=\phi_{i}\left(G^{-1}(\xi)\right) \quad \text { for } A+1 \leq i \leq n-1
\end{aligned}
$$

for $\xi \in \Omega$. It is easy to see that $\Psi_{i}=\psi_{i}$ for $i \geq A+1$. The basis of the function space $\tilde{V}_{h}$ is the set $\left\{\Psi_{i}\right\}_{i=A, \ldots, n-1}$ containing the newly defined functions. One can easily see that $\tilde{V}_{h}$ is a subspace of $V_{h}$ since

$$
\Psi_{A}(\xi)=\sum_{i=0}^{A} \psi_{i}(\xi)
$$

and all other basis functions of $\tilde{V}_{h}$ are contained in $V_{h}$ by definition.

To prove $\tilde{V}_{h} \subset H^{1}$ we need to compute the derivative of $\Phi_{A}$. For $x$ beeing in a neighborhood of $x_{0}=0$ the condition

$$
\sum_{i=0}^{p} \phi_{i}(x)=1
$$

implies

$$
\sum_{i=0}^{A} \phi_{i}(x)=1-\sum_{i=A+1}^{p} \phi_{i}(x),
$$

for $0 \leq A \leq p-1$. Hence

$$
\begin{aligned}
\Phi_{A}^{\prime}(x) & =\sum_{i=0}^{A} \phi_{i}^{\prime}(x)=-\sum_{i=A+1}^{p} \phi_{i}^{\prime}(x) \\
& \sim-\sum_{i=A+1}^{p} C_{i} x^{i-1} \sim-C_{A+1} x^{A} .
\end{aligned}
$$

From this it follows that $\left|\Psi_{A}\right|_{1}^{2}$ exists if and only if

$$
\int_{0}^{1} \frac{\left(-C_{A+1} x^{A}\right)^{2}}{C_{\alpha} P_{\alpha} x^{\alpha-1}} d x
$$

exists. This is equivalent to the existence of $\left|\Psi_{A+1}\right|_{1}^{2}=\left|\psi_{A+1}\right|_{1}^{2}$, which we already considered in Theorem 4.1. Summing up, it follows that $\Psi_{A}$ is in $H^{1}(\Omega)$. Theorem 4.1 states that all other basis functions $\Psi_{i}$, for $i \geq A+1$, are in $H^{1}$, hence $\tilde{V}_{h} \subset H^{1}$.

One can even show that $\tilde{V}_{h}=V_{h} \cap H^{1}$, which is in some sense optimal. The function $\Phi_{A}$ has the property that it is nonnegative and that $\Phi_{A}(0)=1$. So $\Psi_{A}$ is interpolating at the boundary. 
Example The functions $\Phi_{A}$ and $\Psi_{A}$ corresponding to the setting of Example 4.2 are shown in Figure $4\left(\Phi_{A}\right.$ and $\Psi_{A}$ are dashed).
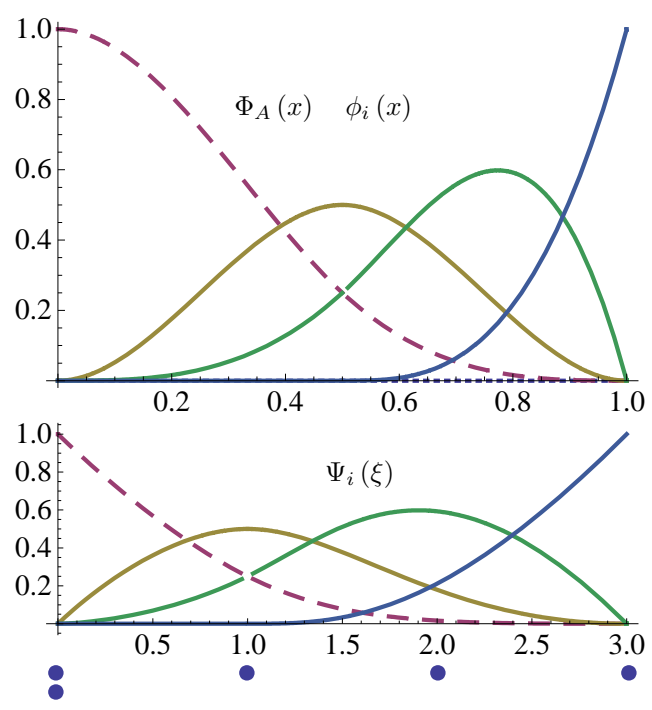

Figure 4: Functions $\Phi_{A}$ and $\phi_{i}$ on $\Omega_{0}$ for $i=2, \ldots, 4, \Psi_{i}$ on $\Omega$ for $i=1, \ldots, 4$ and control points $P_{i}$ for $i=0, \ldots, 4$

The integrands of the stiffness matrix integrals corresponding to the test functions $\Psi_{i}$ are shown in Figure 5, where the graph of the function $\left(\nabla \Psi_{A}(\xi)\right)^{2}$ is a dashed line. In this case all functions are bounded.

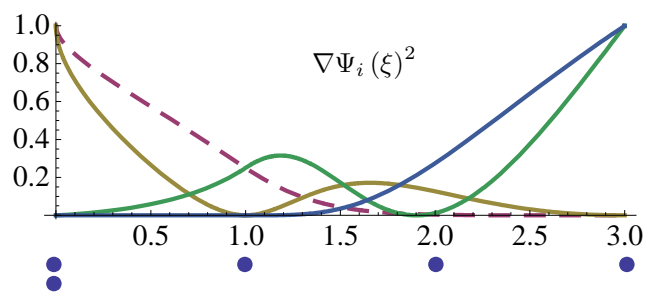

Figure 5: Integrands $\left(\nabla \Psi_{i}(\xi)\right)^{2}$ on $\Omega_{0}$ for $i=1, \ldots, 4$

\subsection{Swept parameterizations of two-dimensional domains}

The results of Theorem 4.1 can be carried over to twodimensional domains described by special parameterizations. We consider a rectangular domain $\Omega$ having a two-dimensional parameterization $\mathbf{G}$ of degree $\mathbf{p}$ with control points $\left(\mathbf{P}_{\mathbf{i}}\right)_{\mathbf{i} \in \mathbb{I}}$. Figure 6 shows an example of a rectangular control point grid which is constructed from the control polygon given by Table 1 (see Example 4.2). In this case the degree is $\mathbf{p}=(3,3)$.

Theorem 4.3 Let $\alpha \in \mathbb{Z}^{+}$, with $2 \leq \alpha \leq p_{1}$. We consider a twodimensional parameterization $\mathbf{G}$ consisting of basis functions $\left(\phi_{\mathbf{i}}\right)_{\mathbf{i} \in \mathbb{I}}$ and control points $\left(\mathbf{P}_{\mathbf{i}}\right)_{\mathbf{i} \in \mathbb{I}}$ and a one-dimensional parameterization $G$ with the control polygon $\mathbf{Q}=\left(Q_{i}\right)_{i \in\left\{0, \ldots, n_{1}-1\right\}}$ fulfilling the assumptions of Theorem 4.1. We assume that the control points fulfill $\mathbf{P}_{(i, j)}=\left(Q_{i}, P_{j}\right)^{T}$ for all $i, j$, where $\left(P_{j}\right)_{j \in\left\{0, \ldots, n_{2}-1\right\}}$ is a strictly monotonically increasing sequence.

Under these assumptions

$$
\psi_{(i, j)} \in H^{1}(\Omega)
$$

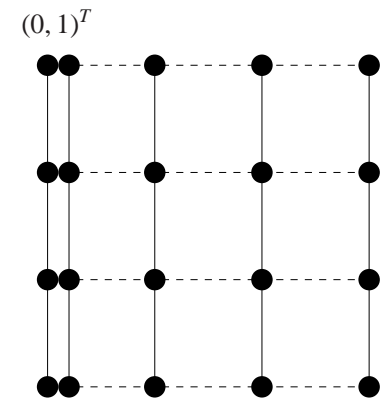

$(0,0)^{T}$

$(1,0)^{T}$

Figure 6: Control points for a swept parameterization

if and only if $0 \leq i \leq\left\lfloor\frac{\alpha}{2}\right\rfloor$.

The proof of this theorem takes advantage of the tensorproduct structure of the bivariate NURBS functions and follows directly from Theorem 4.1. In this situation the function space $V_{h}$ can be modified similar to the one-dimensional case. The framework follows the method introduced in Subsection 4.2.

With the help of Theorem 3.6 the results of Theorem 4.3 can be carried over to more general parameterizations. As an example we consider the following parameterization.

Example 4.4 Let $\mathbf{p}=(2,2)$ and consider a quarter of a circular ring having the parameterization $\hat{\mathbf{G}}$ constructed via the control points given in Figure 7.

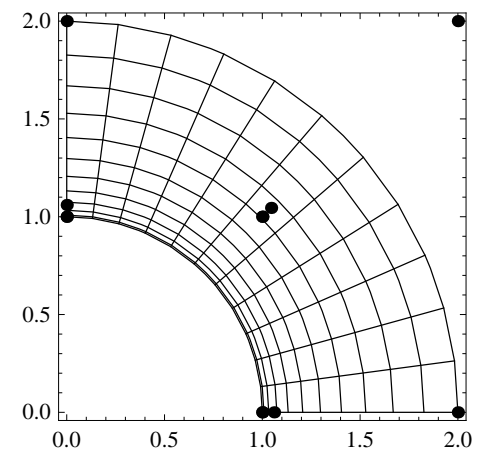

Figure 7: Circular ring and control points

This parameterization of the circular ring is structurally equivalent to the parameterization $\mathbf{G}$ given in Figure 8 . The constants $\underline{\lambda}$ and $\bar{\lambda}$ as defined in Theorem 3.6 can be chosen as $\lambda=1$ and $\overline{\bar{\lambda}}=10.99$.

Figure 9 shows the Jacobian determinant of $\hat{\mathbf{G}}\left(\mathbf{G}^{-1}\right)$. One can show that the determinant is bounded from above and below by positive constants.

Because of the given structure of the parameterization all test functions corresponding to the inner 6 control points are not in $H^{1}$. This follows directly from Theorems 3.6 and 4.3 .

To set up conditions on the control points which guarantee that two parameterizations are structurally equivalent is beyond the scope of this paper.

The basic structure of the previously developed methods for the one-dimensional case can be generalized to various cases of 


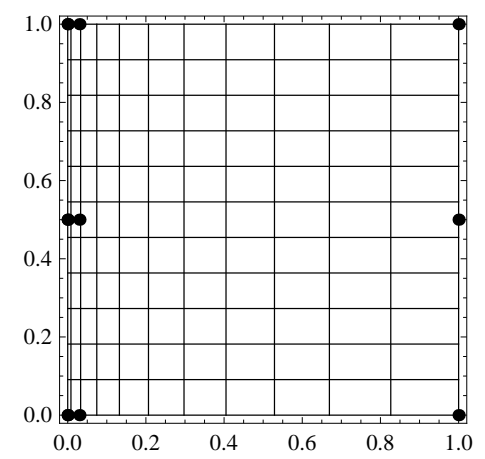

Figure 8: Reference rectangle corresponding to Figure 7

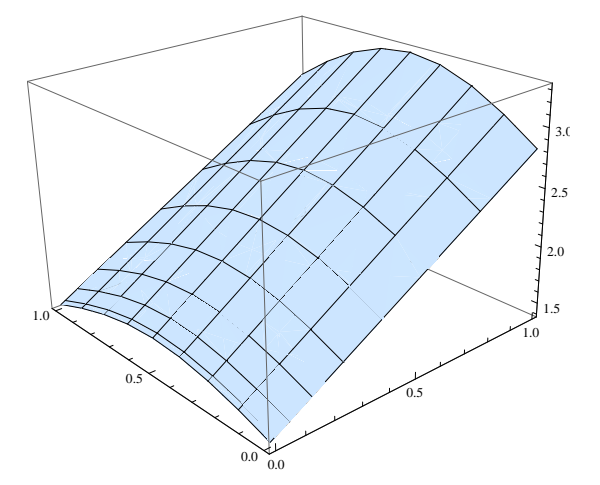

Figure 9: Jacobian determinant $\operatorname{det}(\mathbf{J})$ for the circular ring

two-dimensional parameterizations, which we will study in the next section.

\section{The two-dimensional case}

In the bivariate case we analyze the existence of the stiffness matrix integrals derived from the differential equation (2.1) on a two-dimensional domain $\Omega$, hence the main task is to answer the Existence Problem 2.7.

\subsection{Assumptions}

The functions $\left(\phi_{\mathbf{i}}\right)_{\mathbf{i} \in \mathbb{I}}$ form a NAM family of degree $\mathbf{p}$ near $\mathbf{x}_{0}=\mathbf{O}$, such as NURBS of degree $\mathbf{p}$ with open knot vectors. The parameterization has to be regular everywhere, except for the singularity at $\mathbf{x}_{0}=\mathbf{O}$. There exist different configurations of the control point grid, that lead to such a singularly parameterized domain. In the following we concentrate on two specific cases, which are of practical interest. In the first case (Assumption 5.1) the index set of degeneration is a rectangle and all control points in that set degenerate to one point. In the second case (Assumption 5.2) the index set of degeneration is an $\mathrm{L}$-shape, where all points in the set are collinear.

Assumption 5.1 (Case 1: rectangle) Let $\boldsymbol{\alpha}=\left(\alpha_{1}, \alpha_{2}\right)$ with $\mathbf{1} \leq$ $\alpha \leq$ p. The index set $\mathbb{D}_{\alpha} \subseteq \mathbb{I}$ fulfills

$$
\mathbb{D}_{\alpha}=\{\mathbf{i} \in \mathbb{I}: \mathbf{0} \leq \mathbf{i} \leq \boldsymbol{\alpha}-\mathbf{1}\}
$$

and the control points fulfill

- $\mathbf{P}_{\mathbf{i}}=\mathbf{O}$ for $\mathbf{i} \in \mathbb{D}_{\alpha}$ and

- $\mathbf{P}_{\mathbf{i}} \neq \mathbf{O}$ for $\mathbf{i} \in \mathbb{I} \backslash \mathbb{D}_{\alpha}$.

The triangles

1. $\Delta_{11}=\triangle\left(\mathbf{O}, \mathbf{P}_{\left(\alpha_{1}, 0\right)}, \mathbf{P}_{\left(\alpha_{1}, 1\right)}\right)$

2. $\Delta_{12}=\Delta\left(\mathbf{O}, \mathbf{P}_{\left(\alpha_{1}, 0\right)}, \mathbf{P}_{\left(0, \alpha_{2}\right)}\right)$

3. $\Delta_{13}=\Delta\left(\mathbf{O}, \mathbf{P}_{\left(0, \alpha_{2}\right)}, \mathbf{P}_{\left(1, \alpha_{2}\right)}\right)$

do not degenerate.

An example for the rectangle-case can be seen in Figure 10, where $\alpha_{1}=3$ and $\alpha_{2}=4$. The dots represent double indices $(i, j) \in \mathbb{I}$. The dots inside the bold-lined rectangle represent the set $\mathbb{D}_{\alpha}$.

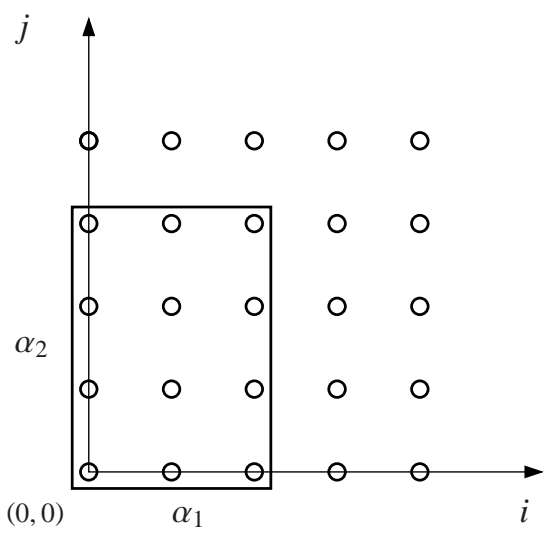

Figure 10: Index set $\mathbb{D}_{\alpha}$ for case 1 (rectangle)

Figure 11 shows an example of a control point grid for bivariate $\mathrm{B}$-Splines of degree $\mathbf{p}=(3,3)$ along with the triangles $\triangle_{11}, \Delta_{12}$ and $\Delta_{13}$. The control points that lie on a common thin continuous or dashed line have a common $i$ - or $j$-index, respectively. Points that are close together in the figure are meant to be equal. This example is a valid Case-1-situation for $\alpha_{1}=2$ and $\alpha_{2}=3$.

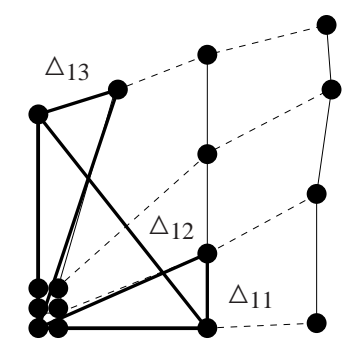

$(0,0)^{T}$

Figure 11: Triangles $\Delta_{11}, \ldots, \Delta_{13}$ for case 1 (with $\alpha_{1}=2, \alpha_{2}=3$ )

Assumption 5.2 (Case 2: L-shape) Let $\boldsymbol{\alpha}=\left(\alpha_{1}, \alpha_{2}\right)$ and $\boldsymbol{\beta}=$ $\left(\beta_{1}, \beta_{2}\right)$ with $\mathbf{1} \leq \boldsymbol{\alpha} \leq \mathbf{p}$ and $\boldsymbol{\alpha} \leq \boldsymbol{\beta} \leq \mathbf{p}+\mathbf{1}$. The two index sets $\mathbb{D}_{\alpha}$ and $\mathbb{D}_{\beta}$ are defined by

$$
\begin{aligned}
\mathbb{D}_{\alpha}= & \left\{(i, 0) \in \mathbb{I}: 0 \leq i \leq \alpha_{1}-1\right\} \\
\cup & \left\{(0, j) \in \mathbb{I}: 0 \leq j \leq \alpha_{2}-1\right\}
\end{aligned}
$$


and

$$
\begin{aligned}
\mathbb{D}_{\beta}= & \left\{(i, 0) \in \mathbb{I}: 0 \leq i \leq \beta_{1}-1\right\} \\
& \cup\left\{(0, j) \in \mathbb{I}: 0 \leq j \leq \beta_{2}-1\right\} .
\end{aligned}
$$

The control points fulfill

- $\mathbf{P}_{\mathbf{i}}=\mathbf{O}$ for $\mathbf{i} \in \mathbb{D}_{\alpha}$,

- $\mathbf{P}_{\mathbf{i}} \neq \mathbf{O}$ for $\mathbf{i} \in \mathbb{I} \backslash \mathbb{D}_{\alpha}$ and

the points $\mathbf{P}_{\mathbf{j}}$ are collinear for $\mathbf{j} \in \mathbb{D}_{\beta}$ and the triangles

1. $\Delta_{21}=\Delta\left(\mathbf{O}, \mathbf{P}_{\left(\alpha_{1}, 0\right)}, \mathbf{P}_{(1,1)}\right)$

2. $\Delta_{22}=\Delta\left(\mathbf{O}, \mathbf{P}_{(1,1)}, \mathbf{P}_{(2,1)}\right)$

3. $\Delta_{23}=\Delta\left(\mathbf{O}, \mathbf{P}_{(1,1)}, \mathbf{P}_{(1,2)}\right)$

4. $\Delta_{24}=\Delta\left(\mathbf{O}, \mathbf{P}_{\left(0, \alpha_{2}\right)}, \mathbf{P}_{(1,1)}\right)$

do not degenerate.

Figure 12 gives an overview of the index sets $\mathbb{D}_{\alpha}$ (bold continuous line) and $\mathbb{D}_{\beta}$ (dashed line) for the $\mathrm{L}$-shape-case, where $\alpha_{1}=3, \alpha_{2}=4, \beta_{1}=4$ and $\beta_{2}=5$.

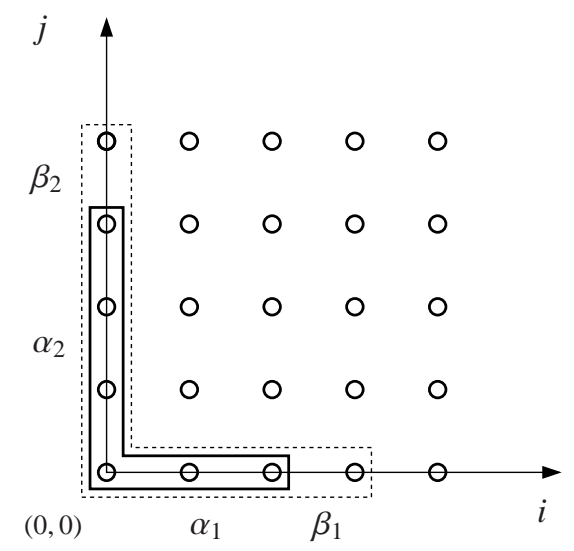

Figure 12: Index sets $\mathbb{D}_{\alpha}$ and $\mathbb{D}_{\beta}$ for case 2 (L-shape)

In Figure 13 we consider B-Splines of degree $\mathbf{p}=(3,3)$. The figure shows an example of a control point grid and triangles $\Delta_{21}, \Delta_{22}, \Delta_{23}$ and $\Delta_{24}$ for a valid Case-2-situation $\left(\alpha_{1}=\alpha_{2}=2\right.$ and $\left.\beta_{1}=\beta_{2}=4\right)$. This figure is to be interpreted similar to Figure 11 .

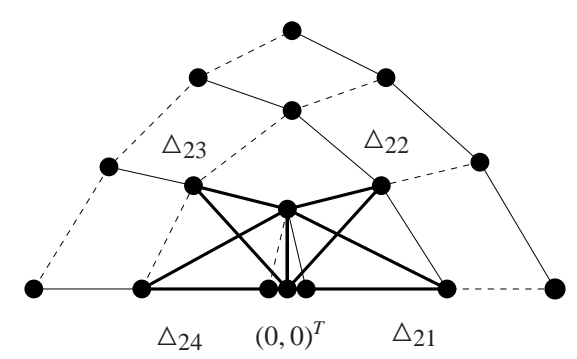

Figure 13: Triangles $\Delta_{21}, \ldots, \Delta_{24}$ for case 2 (with $\alpha_{1}=\alpha_{2}=2$ )

The additional assumptions on the triangles (as depicted in Figure 11 and 13) are sufficient but not necessary to prove the existence of the integrals. It might happen that some of the triangles degenerate, but nevertheless all integrals may still exist.

\subsection{Results}

Similar to the one-dimensional case we have to analyze the square of the $H^{1}$-seminorm of the test functions $\psi_{\mathbf{i}}$. First the integral is transformed to the parameter domain, then the underlying structure of the specific integral is analyzed. Applying the substitution rule to the bivariate integral leads to

$$
\begin{aligned}
\left|\psi_{\mathbf{i}}\right|_{1}^{2} & =\int_{\mathbf{G}\left(\Omega_{0}\right)}\left\|\nabla \psi_{\mathbf{i}}(\boldsymbol{\xi})\right\|^{2} \mathrm{~d} \boldsymbol{\xi} \\
& =\int_{\Omega_{0}}\left\|(\nabla \mathbf{G})^{-T} \nabla \phi_{\mathbf{i}}\right\|^{2} \operatorname{det}(\nabla \mathbf{G}) \mathrm{d} \mathbf{x} \\
& =\int_{\Omega_{0}} \frac{\left\|\operatorname{Cof}(\nabla \mathbf{G}) \nabla \phi_{\mathbf{i}}\right\|^{2}}{\operatorname{det}(\nabla \mathbf{G})} \mathrm{d} \mathbf{x},
\end{aligned}
$$

where $\operatorname{Cof}(\nabla \mathbf{G})$ is the cofactor matrix of the Jacobian matrix $\nabla \mathbf{G}$, with

$$
\operatorname{Cof}\left(\begin{array}{ll}
\frac{\partial G_{1}}{\partial x} & \frac{\partial G_{1}}{\partial y} \\
\frac{\partial G_{2}}{\partial x} & \frac{\partial G_{2}}{\partial y}
\end{array}\right)=\left(\begin{array}{rr}
\frac{\partial G_{2}}{\partial y} & -\frac{\partial G_{2}}{\partial x} \\
-\frac{\partial G_{1}}{\partial y} & \frac{\partial G_{1}}{\partial x}
\end{array}\right) .
$$

Hence the $H^{1}$-seminorm can be written as

$$
\left|\psi_{\mathbf{i}}\right|_{1}^{2}=\int_{\Omega_{0}} \frac{N_{\mathbf{i}}(x, y)}{D(x, y)} \mathrm{d} \mathbf{x}
$$

with the numerator $N_{\mathbf{i}}=\left\|\operatorname{Cof}(\nabla \mathbf{G}) \nabla \phi_{\mathbf{i}}\right\|^{2}$ and the denominator $D=\operatorname{det}(\nabla \mathbf{G})$.

The denominator $D(x, y)$ can be represented by the double sum

$$
D(x, y)=\sum_{\mathbf{k}, \mathbf{l} \in \mathbb{I}} S(\mathbf{k}, \mathbf{l})(x, y) \triangle_{\mathbf{k}, \mathbf{l}},
$$

where

$$
S(\mathbf{k}, \mathbf{l})=\phi_{\mathbf{k}}^{(1)} \phi_{\mathbf{l}}^{(2)}-\phi_{\mathbf{k}}^{(2)} \phi_{\mathbf{l}}^{(1)}
$$

and

$$
\triangle_{\mathbf{k}, \mathbf{l}}=\frac{1}{2}\left(\mathbf{P}_{\mathbf{k}}^{1} \mathbf{P}_{\mathbf{l}}^{2}-\mathbf{P}_{\mathbf{k}}^{2} \mathbf{P}_{\mathbf{l}}^{1}\right) .
$$

Here $\Delta_{\mathbf{k}, \mathbf{l}}$ is the area of the triangle $\Delta\left(\mathbf{O}, \mathbf{P}_{\mathbf{k}}, \mathbf{P}_{\mathbf{l}}\right)$. The numerator $N_{\mathbf{i}}(x, y)$ fulfills

$$
N_{\mathbf{i}}(x, y)=\sum_{\mathbf{k}, \mathbf{l} \in \mathbb{I}} S(\mathbf{k}, \mathbf{i}) S(\mathbf{l}, \mathbf{i})\left\langle\mathbf{P}_{\mathbf{k}}, \mathbf{P}_{\mathbf{l}}\right\rangle .
$$

Using this representation we can prove existence results for the model cases described in Assumptions 5.1 and 5.2.

Theorem 5.3 If Assumption 5.1 (Case 1) or Assumption 5.2 (Case 2) is fulfilled, then all test functions $\psi_{\mathbf{i}}$, for $\mathbf{i} \in \mathbb{I}$, are in $H^{1}(\Omega)$.

The core idea of the proof is to analyze the asymptotic behavior of the denominator and of the numerator separately. Each function depends on the control points $\mathbf{P}_{\mathbf{i}}$ and on the basis functions $\phi_{\mathbf{i}}$, therefore the asymptotic behavior of both denominator and numerator can be computed explicitly under the assumptions of Section 3. Finally the integrability condition (Theorem 3.2) leads to a rational function that can be integrated over the parameter domain. 
Proof of Theorem 5.3. We prove that the square of the $H^{1}$ seminorm of the function $\psi_{\mathbf{i}}$ is well-defined. The denominator has a double sum structure, where we sum over the index set $\mathbb{I}$ twice. The bivariate function $D[\mathbb{S}, \mathbb{T}]$ is defined by

$$
D[\mathbb{S}, \mathbb{T}]=\sum_{\mathbf{k} \in \mathbb{S}} \sum_{\mathbf{l} \in \mathbb{T}} S(\mathbf{k}, \mathbf{l}) \triangle_{\mathbf{k}, \mathbf{l}},
$$

which is a partial sum of the denominator over some smaller index sets $\mathbb{S}$ and $\mathbb{T}$. It is clear, that $D(x, y)=D[\mathbb{I}, \mathbb{I}](x, y)$ by definition. The asymptotic behavior of $D$ near $\mathbf{x}_{0}$ depends only on some of the basis functions. Hence we can define a proper subset $\mathbb{K}$ of $\mathbb{I}$, such that $D[\mathbb{I}, \mathbb{I}] \sim D[\mathbb{K}, \mathbb{K}]$ and $|\mathbb{K}| \ll|\mathbb{I}|$. The set $\mathbb{K}$ is of course depending on the degeneration of the control grid.

It is obvious that $\Delta_{\mathbf{k}, \mathbf{l}}=\Delta_{\mathbf{l}, \mathbf{k}}=0$ for all $\mathbf{k} \in \mathbb{D}_{\alpha}$ and for all $\mathbf{l} \in \mathbb{I}$, since all control points corresponding to $\mathbb{D}_{\alpha}$ are equal to O. Hence $D[\mathbb{I}, \mathbb{I}]=D[\mathbb{J}, \mathbb{J}]$ for $\mathbb{J}=\mathbb{I} \backslash \mathbb{D}_{\alpha}$.

The specific setting of $\mathbb{K}$ is

$$
\mathbb{K}=\left\{\left(\alpha_{1}, 0\right),\left(\alpha_{1}, 1\right),\left(0, \alpha_{2}\right),\left(1, \alpha_{2}\right)\right\}
$$

in Case 1 and

$$
\mathbb{K}=\left\{\left(\alpha_{1}, 0\right),\left(0, \alpha_{2}\right),(1,1),(1,2),(2,1)\right\}
$$

in Case 2. Now the asymptotic behavior of $D[\mathbb{K}, \mathbb{K}]$ can be computed explicitly and follows directly from the Assumptions 5.1 and 5.2, respectively, and from the results derived from Definition 3.4. In Case 1 there exist constants $K_{1}, K_{2}, K_{3}>0$ such that

$$
D[\mathbb{K}, \mathbb{K}] \sim K_{1} x^{2 \alpha_{1}-1}+K_{2} x^{\alpha_{1}-1} y^{\alpha_{2}-1}+K_{3} y^{2 \alpha_{2}-1}
$$

and in Case 2 there exist constants $K_{1}, K_{2}, K_{3}, K_{4}>0$ such that

$$
D[\mathbb{K}, \mathbb{K}] \sim K_{1} x^{\alpha_{1}}+K_{2} x^{2} y+K_{3} x y^{2}+K_{4} y^{\alpha_{2}} .
$$

Note that $K_{i}>0$ if $\Delta_{1 i}$ or $\Delta_{2 i}$ does not degenerate, respectively. For this representation of $D[\mathbb{K}, \mathbb{K}]$ one can easily show via the equations (8), that in both cases

$$
\left|\phi_{\mathbf{i}}^{(1)} \phi_{\mathbf{j}}^{(2)}\right|,\left|\phi_{\mathbf{i}}^{(2)} \phi_{\mathbf{j}}^{(1)}\right| \in \mathrm{o}(D[\mathbb{K}, \mathbb{K}]),
$$

for $\mathbf{i} \in \mathbb{J}$ and $\mathbf{j} \in \mathbb{J} \backslash \mathbb{K}$. From this it follows immediately that

$$
D[\mathbb{J} \backslash \mathbb{K}, \mathbb{J}]=D[\mathbb{J}, \mathbb{d} \backslash \mathbb{K}] \in \mathrm{o}(D[\mathbb{K}, \mathbb{K}]),
$$

hence $D[\mathbb{I}, \mathbb{I}]=D[\mathbb{J}, \mathbb{J}] \sim D[\mathbb{K}, \mathbb{K}]$.

Some preliminary considerations are needed to analyze the behavior of the numerator $N_{\mathrm{i}}$. From Assumptions 5.1 and 5.2 it follows that $\left\langle\mathbf{P}_{\mathbf{k}}, \mathbf{P}_{\mathbf{l}}\right\rangle=0$ for $\mathbf{k} \in \mathbb{D}_{\alpha}$ or $\mathbf{l} \in \mathbb{D}_{\alpha}$. The derivatives $\phi_{\mathbf{i}}^{(l)}$ and the scalar products $\left\langle\mathbf{P}_{\mathbf{k}}, \mathbf{P}_{\mathbf{l}}\right\rangle$ can be bounded from above. There exist real constants $C_{1}, C_{2}>0$ such that

$$
\left|\phi_{\mathbf{i}}^{(l)}\right| \leq C_{1}
$$

and

$$
\left\langle\mathbf{P}_{\mathbf{k}}, \mathbf{P}_{\mathbf{l}}\right\rangle \leq C_{2}
$$

for $\mathbf{k}, \mathbf{l} \in \mathbb{J}$. It follows from equation (11) and the triangular inequality that

$$
\left|N_{\mathbf{i}}\right| \leq C_{1} C_{2} \sum_{\mathbf{k}, \mathbf{l} \in \mathbb{J}}\left(\left|\phi_{\mathbf{k}}^{(1)}\right|+\left|\phi_{\mathbf{k}}^{(2)}\right|\right)\left(\left|\phi_{\mathbf{l}}^{(1)}\right|+\left|\phi_{\mathbf{I}}^{(2)}\right|\right),
$$

which is equivalent to

$$
N_{\mathbf{i}} \in \mathrm{O}\left(\left(\sum_{\mathbf{k} \in \mathbb{J}}\left|\phi_{\mathbf{k}}^{(1)}\right|+\sum_{\mathbf{k} \in \mathbb{J}}\left|\phi_{\mathbf{k}}^{(2)}\right|\right)^{2}\right) .
$$

Using this representation, it is easy to show that in Case 1 the numerator $N_{\mathbf{i}}$ fulfills

$$
N_{\mathbf{i}} \in \mathrm{O}\left(\left(x^{\alpha_{1}-1}+y^{\alpha_{2}-1}\right)^{2}\right)
$$

and in Case 2 it fulfills

$$
N_{\mathbf{i}} \in \mathrm{O}\left((x+y)^{2}\right)
$$

if $\alpha_{1}>1$ and $\alpha_{2}>1$ or

$$
N_{\mathbf{i}} \in \mathrm{O}(1)
$$

if either $\alpha_{1}=1$ or $\alpha_{2}=1$.

Since the parameterization is regular in every point different from $\mathbf{x}_{0}$, it follows from the integrability condition (Theorem 3.2) that $\left|\psi_{\mathbf{i}}\right|_{1}^{2}<\infty$ if, in Case 1 , the function

$$
\frac{\left(x^{\alpha_{1}-1}+y^{\alpha_{2}-1}\right)^{2}}{K_{1} x^{2 \alpha_{1}-1}+K_{2} x^{\alpha_{1}-1} y^{\alpha_{2}-1}+K_{3} y^{2 \alpha_{2}-1}}
$$

is integrable on the unit square. In Case 2 the same is true, if

$$
\frac{(x+y)^{2}}{K_{1} x^{\alpha_{1}}+K_{2} x^{2} y+K_{3} x y^{2}+K_{4} y^{\alpha_{2}}},
$$

for $\alpha_{1}, \alpha_{2}>1$,

$$
\frac{1}{K_{1} x+K_{4} y^{\alpha_{2}}},
$$

for $\alpha_{1}=1$, or

$$
\frac{1}{K_{1} x^{\alpha_{1}}+K_{4} y}
$$

for $\alpha_{2}=1$, is integrable on the unit square. For the two latter subcases it is not necessary that the triangles $\Delta_{22}$ and $\Delta_{23}$ do not degenerate. All the conditions are fulfilled for arbitrary $\alpha$, which can be shown easily using elementary calculus, hence all test functions $\psi_{\mathbf{i}}$ have well-defined $H^{1}$-seminorms, so they are in $H^{1}(\Omega)$.

Similar existence results can be derived for other types of singularities (e.g. other sets of degeneration). We restrict ourselves to the two model cases, because the two considered cases cover most of the examples which are of practical interest (of course under the assumption that only finitely many singularities at the vertices of the rational segments of the spline parameterization are present). Both cases, the rectangular case as well as the Lshape case, lead to the existence of all stiffness matrix integrals for the standard test functions. The only condition on the basis functions is that they have to form an NAM family of degree $\mathbf{p}$, such as NURBS. 


\subsection{Swept parameterizations of three-dimensional domains}

Similar to Theorem 4.3 we can derive existence results for parameterizations of special three-dimensional domains, which are constructed from planar patches. For further information on the generation of swept volume parameterizations see e.g. [31].

We consider a parameterization $\mathbf{G}^{[2]}$ of the two-dimensional domain $\Omega^{[2]}$ consisting of basis functions $\left(\phi_{\mathbf{j}}(x, y)\right)_{\mathbf{j} \in \mathbb{J}}$ and control points $\left(\mathbf{Q}_{\mathbf{j}}\right)_{\mathbf{j} \in \mathbb{J}}$. The three-dimensional domain $\Omega^{[3]}$ has a parameterization $\mathbf{G}^{[3]}$ with basis functions $\left(\phi_{(i, j)}(x, y) \phi_{k}(z)\right)_{(i, j, k) \in \mathbb{I}}$ and control points $\left(\mathbf{P}_{\mathbf{i}}\right)_{\mathbf{i} \in \mathbb{I}}$ constructed from $\left(\mathbf{Q}_{\mathbf{j}}\right)_{\mathbf{j} \in \mathbb{J}}$ (as in Theorem 5.4). Here we consider the index sets

$$
\mathbb{I}=\left\{\mathbf{i}=(i, j, k) \in \mathbb{N}^{3}: \mathbf{0} \leq \mathbf{i} \leq\left(n_{1}, n_{2}, n_{3}\right)-\mathbf{1}\right\}
$$

and

$$
\mathbb{J}=\left\{\mathbf{j}=(i, j) \in \mathbb{N}^{2}: \mathbf{0} \leq \mathbf{j} \leq\left(n_{1}, n_{2}\right)-\mathbf{1}\right\} .
$$

Figure 14 shows a volumetric parameterization of a cylindric domain. In this case the underlying two-dimensional patch is the circle from Example 6.2.

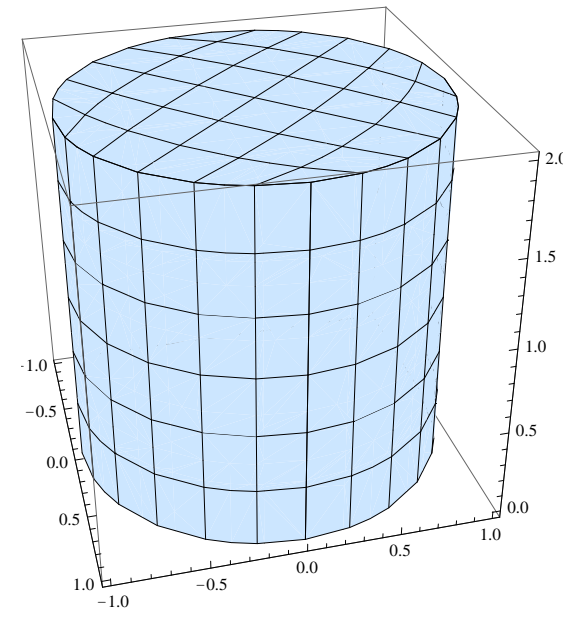

Figure 14: Cylindric parameterization

Theorem 5.4 Let $\Omega^{[3]}$ be a volume constructed from the twodimensional domain $\Omega^{[2]}$, i.e. for $\mathbf{i} \in \mathbb{I}$ the control point $\mathbf{P}_{\mathbf{i}}$ fulfills $\mathbf{P}_{(i, j, k)}=\left(Q_{(i, j)}^{1}, Q_{(i, j)}^{2}, P_{k}\right)^{T}$, where $\left(P_{k}\right)_{k \in\left\{0, \ldots, n_{3}-1\right\}}$ is a strictly monotonically increasing sequence. Each trivariate test function $\psi_{(i, j, k)}$ fulfills

$$
\psi_{(i, j, k)}=\phi_{(i, j)} \phi_{k} \circ\left(\mathbf{G}^{[3]}\right)^{-1} \in H^{1}\left(\Omega^{[3]}\right)
$$

if and only if the bivariate test function $\psi_{(i, j)}$ fulfills

$$
\psi_{(i, j)}=\phi_{(i, j)} \circ\left(\mathbf{G}^{[2]}\right)^{-1} \in H^{1}\left(\Omega^{[2]}\right) .
$$

This theorem states existence results for cylindric domains. It can be applied to more general domains using Theorem 3.6. In combination with the examples from Subsection 6.2 one can especially derive results for circular cylinders or tori. Figure
15 shows the quarter of a torus. The parameterization of the torus is structurally equivalent to the cylindric parameterization shown in Figure 14. In this case all test functions on the torus are in $H^{1}$. This follows directly from Theorems 3.6 and 5.4 and the existence results for Example 6.2.

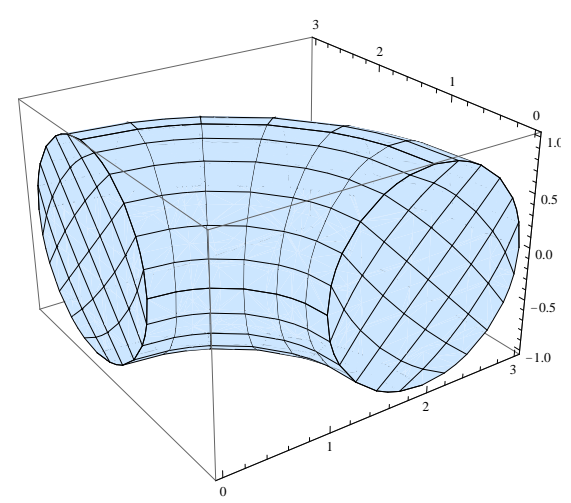

Figure 15: Quarter of a torus

\section{Case studies}

There may occur different kinds of singularities, not covered by the model cases, which lead to the non-existence of some of the stiffness matrix integrals. In those cases we can modify the set of basis functions to be able to guarantee that all new basis functions are in $H^{1}(\Omega)$. In this section we present and compare different ways to parameterize basic geometric objects. In Subsection 6.1 we parameterize a triangle and in Subsection 6.2 we parameterize the unit disc.

\subsection{Triangular domains}

A parameterization of a triangle as a tensor-product patch can be obtained by collapsing one edge into a single point. This approach is not covered by the previously defined cases (Assumptions 5.1 and 5.2). As a model case, we analyze a patch of degree $\mathbf{p}=\left(p_{1}, p_{2}\right)$ with knot vectors

$$
\begin{aligned}
& \theta_{1}=(0, \ldots, 0,1, \ldots, 1) \in \mathbb{R}^{2 p_{1}+2}, \\
& \theta_{2}=(0, \ldots, 0,1, \ldots, 1) \in \mathbb{R}^{2 p_{2}+2},
\end{aligned}
$$

and control points

$$
\mathbf{P}_{(i, j)}=\left(\frac{i}{p_{1}}, \frac{i}{p_{1}} \cdot \frac{j}{p_{2}}\right) .
$$

The parameterization fulfills

$$
\mathbf{G}(x, y)=(x, x y),
$$

where $\operatorname{det} \nabla \mathbf{G}=x$ and

$$
\operatorname{Cof}(\nabla \mathbf{G})(x, y)=\left(\begin{array}{rr}
x & -y \\
0 & 1
\end{array}\right) .
$$




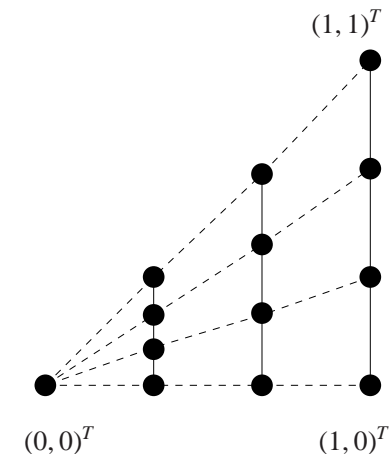

Figure 16: Control points for the triangle case (with $p_{1}=p_{2}=3$ )

Figure 16 shows the control point grid for $\mathbf{p}=(3,3)$ and the three extremal points $(0,0)^{T},(1,0)^{T}$ and $(1,1)^{T}$.

One can show that for each $j \in\left\{0, \ldots, p_{2}\right\}$ exists a $c>0$, an $\epsilon>0$ and an interval $] y_{1}, y_{2}[\subset] 0,1[$ such that

$$
c<\left\|\left(\begin{array}{rr}
x & -y \\
0 & 1
\end{array}\right) \nabla \phi_{(0, j)}(x, y)\right\|^{2}
$$

for all $x<\epsilon$ and for all $y \in] y_{1}, y_{2}[$. Using this we get

$$
\left|\psi_{(0, j)}\right|_{1}^{2}>\int_{\delta}^{\epsilon} \int_{y_{1}}^{y_{2}} \frac{c}{x} \mathrm{~d} y \mathrm{~d} x \underset{\delta \rightarrow 0}{\longrightarrow} \infty,
$$

hence $\psi_{(0, j)}$ is not in $H^{1}(\Omega)$. Thus, we need to modify these functions, in order to make them suitable for the Isogeometric Analysis.

For $i>0$ the gradient of $\phi_{(i, j)}$ is of the form

$$
\nabla \phi_{(i, j)}=\left(Q_{1}(x, y), x^{i} Q_{2}(x, y)\right)^{T}
$$

where $Q_{1}(x, y)$ and $Q_{2}(x, y)$ are some polynomials. Therefore

$$
\left\|\left(\begin{array}{rr}
x & -y \\
0 & 1
\end{array}\right) \nabla \phi_{(i, j)}(x, y)\right\|^{2}=x^{2}(Q(x, y))^{2},
$$

where $Q(x, y)$ is some polynomial. From this it follows directly that the $H^{1}$-seminorm of $\psi_{(i, j)}$ exists for $i>0$, since the denominator of the integrand $\operatorname{det} \nabla \mathbf{G}(x, y)=x$ cancels out.

Using again the approach taken in the examples in [1], we modify the set of basis functions such that all considered functions are in $H^{1}$. We introduce an approach similar to the method in the one-dimensional case. One can easily see that the sum of the first row of basis functions

$$
\boldsymbol{\varphi}_{0}(x, y)=\sum_{j=0}^{p_{2}} \phi_{(0, j)}(x, y)
$$

is equal to the 0 -th univariate $\mathrm{B}$-spline function of degree $p_{1}$, i.e.

$$
\varphi_{0}(x, y)=B_{0, p_{1}}(x) \quad \forall(x, y) \in \Omega_{0} .
$$

For the new test function $\psi_{0}$ defined by $\psi_{0}=\varphi_{0} \circ \mathbf{G}^{-1}$ it can be shown that

$$
\begin{aligned}
\left|\psi_{0}\right|_{H^{1}}^{2} & =\int_{\Omega_{0}} \frac{x^{2}\left(\frac{\partial \varphi_{0}(x, y)}{\partial x}\right)^{2}}{x} \mathrm{~d} \mathbf{x} \\
& =\int_{\Omega_{0}} x\left(B_{0, p_{1}}^{\prime}(x)\right)^{2} \mathrm{~d} \mathbf{x}<\infty .
\end{aligned}
$$

Using $\psi_{0}$ we construct a new function space $\tilde{V}_{h}$, as the space spanned by the basis $\left\{\psi_{0}\right\} \cup\left\{\psi_{(i, j)}\right\}_{i=1, \ldots, p_{1}}^{j=0, \ldots, p_{2}}$. Since all test functions are in $H^{1}$ we get $\tilde{V}_{h} \subset H^{1}$. One can again show that $\tilde{V}_{h}=H^{1} \cap V_{h}$. The presented method can be generalized to other instances of singularly parameterized domains.

However, the L-shape case as described in Assumption 5.2 can also be used to parameterize a triangular domain. Figure 10 shows one example of a control point grid of a triangular domain with curved boundary. Theorem 5.3 states that in this case all test functions are in $H^{1}(\Omega)$. So this strategy to parameterize a triangle does not require further manipulation of the function space $V_{h}(\Omega)$.

\subsection{Circular domains}

The methods and results from Subsection 5.2 can be applied when parameterizing the interior of the unit circle. Here we compare two different parameterizations. Both methods have been presented in [22]. The first example has a polar coordinate structure.

Example 6.1 Consider a NURBS parameterization of degree $\mathbf{p}=(1,2)$ with knot vectors $\theta_{1}=(0,0,1,1)$ and $\theta_{2}=$ $\left(0,0,0, \frac{1}{4}, \frac{1}{4}, \frac{1}{2}, \frac{1}{2}, \frac{3}{4}, \frac{3}{4}, 1,1,1\right)$. The control points and weights are given in Table 2 .

\begin{tabular}{lrr}
\hline $\mathbf{P}_{(i, j)}, w_{(i, j)}$ & $i=0$ & $i=1$ \\
\hline$j=0$ & $(0,0)^{T}, 1$ & $(1,0)^{T}, 1$ \\
$j=1$ & $(0,0)^{T}, \frac{1}{\sqrt{2}}$ & $(1,1)^{T}, \frac{1}{\sqrt{2}}$ \\
$j=2$ & $(0,0)^{T}, 1$ & $(0,1)^{T}, 1$ \\
$j=3$ & $(0,0)^{T}, \frac{1}{\sqrt{2}}$ & $(-1,1)^{T}, \frac{1}{\sqrt{2}}$ \\
$j=4$ & $(0,0)^{T}, 1$ & $(-1,0)^{T}, 1$ \\
$j=5$ & $(0,0)^{T}, \frac{1}{\sqrt{2}}$ & $(-1,-1)^{T}, \frac{1}{\sqrt{2}}$ \\
$j=6$ & $(0,0)^{T}, 1$ & $(0,-1)^{T}, 1$ \\
$j=7$ & $(0,0)^{T}, \frac{1}{\sqrt{2}}$ & $(1,-1)^{T}, \frac{1}{\sqrt{2}}$ \\
$j=8$ & $(0,0)^{T}, 1$ & $(1,0)^{T}, 1$ \\
\hline
\end{tabular}

Table 2: Control points and weights for Example 6.1

Figure 17 shows the control points and Figure 18 shows the parameterization for Example 6.1. In Figure 17 the control points that lie on a common continuous or dashed line have a common $i$ - or $j$-index, respectively. In this situation all control points with $i=0$ degenerate to the origin. This leads to a singular parameterization with $\nabla \mathbf{G}(0, y)=0$ for $0 \leq y \leq 1$. Basically, this singularity is similar to the triangular parameterization from Subsection 6.1. 


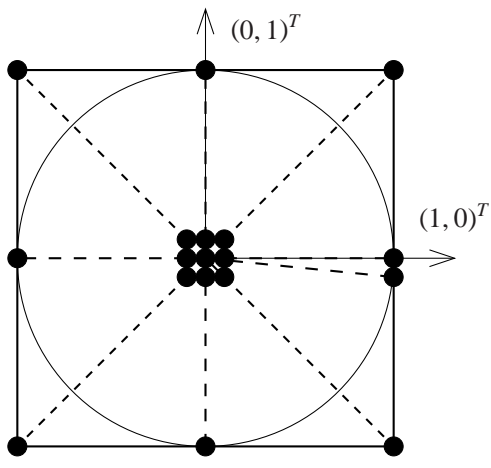

Figure 17: Control points for Example 6.1

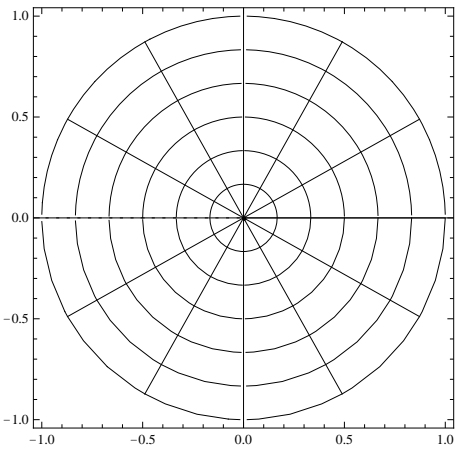

Figure 18: Parameterization for Example 6.1

Among the 18 test functions $\psi_{(i, j)}$ only those with $i=1$ are in $H^{1}(\Omega)$. The basis of $V_{h}$ has to be modified in order to achieve $\tilde{V}_{h}(\Omega) \subseteq C^{0}(\Omega) \cap H^{1}(\Omega)$. This results in a total number of 9 basis functions for $\tilde{V}_{h}$ after modification, since all $\psi_{(0, j)}$ for $0 \leq j \leq 8$ as well as $\psi_{(1,0)}$ and $\psi_{(1,8)}$ have to be summed up.

The second possibility to parameterize a circular domain is given in Example 6.2. It has a more Cartesian-like structure than Example 6.1.

Example 6.2 Consider a NURBS parameterization of degree $\mathbf{p}=(2,2)$ with knot vectors $\theta_{1}=\theta_{2}=(0,0,0,1,1,1)$. The control points and weights are given in Table 3.

\begin{tabular}{lrrr}
\hline $\mathbf{P}_{(i, j)}, w_{(i, j)}$ & $i=0$ & $i=1$ & $i=2$ \\
\hline$j=0$ & $(-1,0)^{T}, \quad 1$ & $(-1,-1)^{T}, \frac{1}{\sqrt{2}}$ & $(0,-1)^{T}, \quad 1$ \\
$j=1$ & $(-1,1)^{T}, \frac{1}{\sqrt{2}}$ & $(0,0)^{T}, \quad 1$ & $(1,-1)^{T}, \frac{1}{\sqrt{2}}$ \\
$j=2$ & $(0,1)^{T}, \quad 1$ & $(1,1)^{T}, \frac{1}{\sqrt{2}}$ & $(1,0)^{T}, \quad 1$ \\
\hline
\end{tabular}

Table 3: Control points and weights for Example 6.2

The control points and the parameterization corresponding to Example 6.2 are shown in Figures 19 and 20. Similarly to Figure 17 the control points in Figure 19 which lie on a common continuous or dashed line have a common $i$ - or $j$-index.

Here we have four singularities at the parameter values $\mathbf{x}_{1}=$ $(0,0)^{T}, \mathbf{x}_{2}=(0,1)^{T}, \mathbf{x}_{3}=(1,1)^{T}$ and $\mathbf{x}_{4}=(1,0)^{T}$. From Theorem 5.3 it follows that all test functions are in $H^{1}$, since the parameterization is symmetric with respect to the singular points.

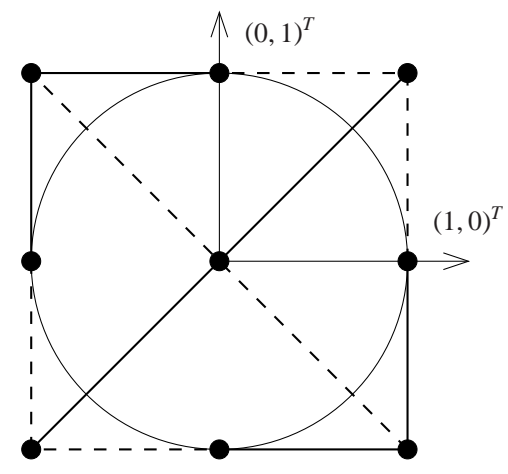

Figure 19: Control points for Example 6.2

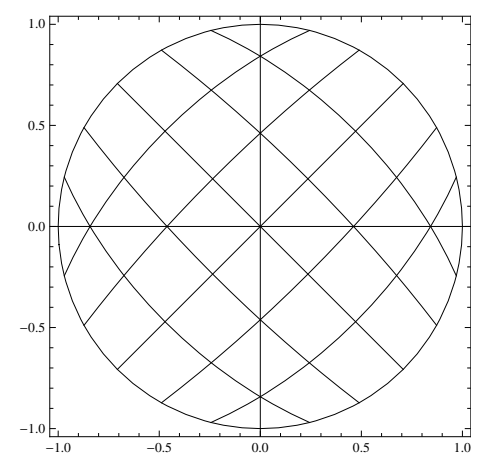

Figure 20: Parameterization for Example 6.2

Hence the function space $V_{h}$ is spanned by 9 basis functions and fulfills $V_{h}(\Omega) \subseteq C^{\infty}(\Omega) \cap H^{1}(\Omega)$.

In Example 6.2 the basis functions do not require a modification but are even in $C^{\infty}$. In Example 6.1 the test function $\Psi_{0}$ which corresponds to the origin is interpolating at the origin, i.e. $\Psi_{0}(0,0)=1$, but has no well-defined gradient in this point. In Example 6.2 the corresponding test function $\psi_{(1,1)}$ is smooth everywhere but fulfills $\psi_{(1,1)}(0,0)<1$, so it is not interpolating. When restricted to the boundary of $\Omega$, the values of the basis functions corresponding to the 8 outer control points are the same for both parameterizations. We highly recommend to use the parameterization given in Example 6.2 rather than the parameterization from Example 6.1, since it is more concise and does not require further manipulation of the basis functions.

\section{Conclusions}

We considered the isogeometric approach for the numerical discretization of a general second order elliptic partial differential equation in the one- and two-dimensional case. We assumed that the control points degenerate such that the parameterization possesses a singular point in one corner of the parameter domain. This situation can occur when some of the control points near the boundary are equal or collinear.

In the one-dimensional case we showed that if the first $\alpha$ control points are equal, then the first $\lfloor\alpha / 2\rfloor+1$ test functions are not in $H^{1}$. We showed the existence of all stiffness matrix integrals for two special (but practically interesting) cases in the 
two-dimensional case. If some test functions are not in $H^{1}$ because of a singularity, one may show that their sum is an $H^{1}$ function and use it as a test function instead. This was demonstrated in the one-dimensional case and for a parameterization of a triangle with a collapsing edge. A more general study will be a topic for future research.

Note that the results of our paper can be extended to parameterizations with several singular points, provided that the singularities occur at the vertices of the polynomial or rational segments. Singular points in the interior of edges, however, require a separate analysis, which should be analyzed in the future.

Strong distortions of the control point grid may lead to parameterizations that contain many singular or almost singular points. By almost singular points we understand points, where the determinant of the parameterization gradient is close to 0 . There may occur severe singularities, that lead to the nonexistence of all stiffness matrix integrals in one specific NURBS patch. One area of future research will be to characterize other singularities and to analyze the asymptotic behavior of the integrand functions. The goal will be to prove qualitative existence results as well as quantitative results concerning the condition number of the stiffness matrix or the approximation power of the discretized space.

One should also consider other differential equations, which lead to function spaces different from $H^{1}(\Omega)$. An example would be the space $H(\operatorname{div} ; \Omega)$ which is defined as the subspace of $L^{2}(\Omega)$, where the divergence of any function is an $L^{2}$ function as well. It may happen, that NURBS test functions are contained in $H(\operatorname{div} ; \Omega)$ but not in $H^{1}(\Omega)$.

Of particular interest is also the extension to higher dimensions. Especially for three-dimensional NURBS-based representations, which are widely used in technical applications, existence results are of importance.

\section{Acknowledgements}

The first author was supported by the government of Upper Austria through the Doctoral Program "Computational Mathematics" at the Johannes Kepler University of Linz.

\section{Appendix}

Proof of Theorem 3.2. Let $0<\epsilon<1$ and $C>0$. We know from the asymptotic behavior of $f$ and $g$ that there exists a $\delta>0$ such that $(1-\epsilon) \bar{g}(\mathbf{x})<g(\mathbf{x})$ and $f(\mathbf{x}) \leq C \bar{f}(\mathbf{x})$ for all $\mathbf{x} \in$ $\Omega_{0} \cap U_{\delta}\left(\mathbf{x}_{0}\right)$. The integral can be split, such that

$$
\begin{aligned}
\int_{\Omega_{0}} \frac{f(\mathbf{x})}{g(\mathbf{x})} \mathrm{d} \mathbf{x} & =\int_{\Omega_{0} \cap U_{\delta}\left(\mathbf{x}_{0}\right)} \frac{f(\mathbf{x})}{g(\mathbf{x})} \mathrm{d} \mathbf{x} \\
& +\int_{\Omega_{0} \backslash U_{\delta}\left(\mathbf{x}_{0}\right)} \frac{f(\mathbf{x})}{g(\mathbf{x})} \mathrm{d} \mathbf{x},
\end{aligned}
$$

where

$$
\int_{\Omega_{0} \cap U_{\delta}\left(\mathbf{x}_{0}\right)} \frac{f(\mathbf{x})}{g(\mathbf{x})} \mathrm{d} \mathbf{x}<\frac{1}{1-\epsilon} \int_{\Omega_{0} \cap U_{\delta}\left(\mathbf{x}_{0}\right)} \frac{f(\mathbf{x})}{\bar{g}(\mathbf{x})} \mathrm{d} \mathbf{x}
$$

and

$$
\int_{\Omega_{0} \cap U_{\delta}\left(\mathbf{x}_{0}\right)} \frac{f(\mathbf{x})}{\bar{g}(\mathbf{x})} \mathrm{d} \mathbf{x} \leq C \int_{\Omega_{0} \cap U_{\delta}\left(\mathbf{x}_{0}\right)} \frac{\bar{f}(\mathbf{x})}{\bar{g}(\mathbf{x})} \mathrm{d} \mathbf{x} .
$$

Since $g$ is continuous, it is bounded from below by some constant $c>0$, so

$$
\int_{\Omega_{0} \backslash U_{\delta}\left(\mathbf{x}_{0}\right)} \frac{f(\mathbf{x})}{g(\mathbf{x})} \mathrm{d} \mathbf{x} \leq M .
$$

From the assumption

$$
\int_{\Omega_{0}} \frac{\bar{f}(\mathbf{x})}{\bar{g}(\mathbf{x})} \mathrm{d} \mathbf{x}=D<\infty
$$

it follows

$$
\int_{\Omega_{0}} \frac{f(\mathbf{x})}{g(\mathbf{x})} \mathrm{d} \mathbf{x}<M+\frac{C D}{1-\epsilon}<\infty .
$$

\section{References}

[1] T. Hughes, J. Cottrell, Y. Bazilevs, Isogeometric analysis: CAD, finite elements, NURBS, exact geometry and mesh refinement, Computer Methods in Applied Mechanics and Engineering194(2005) 4135 -95.

[2] Y. Bazilevs, I. Akkerman, Large eddy simulation of turbulent TaylorCouette flow using isogeometric analysis and the residual-based variational multiscale method, Journal of Computational Physics229(2010) $3402-14$.

[3] H. Gomez, T. Hughes, X. Nogueira, V. Calo, Isogeometric analysis of the isothermal Navier-Stokes-Korteweg equations, Computer Methods in Applied Mechanics and Engineering199(2010) 1828 -40.

[4] Y. Zhang, Y. Bazilevs, S. Goswami, C. Bajaj, T. Hughes, Patient-specific vascular NURBS modeling for isogeometric analysis of blood flow, Computer Methods in Applied Mechanics and Engineering196(2007) 2943 59.

[5] A. Buffa, G. Sangalli, R. Vázquez, Isogeometric analysis in electromagnetics: B-splines approximation, Computer Methods in Applied Mechanics and Engineering199(2010) 1143-52.

[6] D. Benson, Y. Bazilevs, M. Hsu, T. Hughes, Isogeometric shell analysis: The Reissner-Mindlin shell, Computer Methods in Applied Mechanics and Engineering199(2010) 276-89.

[7] J. Kiendl, Y. Bazilevs, M.-C. Hsu, R. Wüchner, K.-U. Bletzinger, The bending strip method for isogeometric analysis of Kirchhoff-Love shell structures comprised of multiple patches, Computer Methods in Applied Mechanics and Engineering199(2010) 2403 -16.

[8] J. Cottrell, A. Reali, Y. Bazilevs, T. Hughes, Isogeometric analysis of structural vibrations, Computer Methods in Applied Mechanics and Engineering195(2006) 5257 -96.

[9] X. Qian, Full analytical sensitivities in NURBS based isogeometric shape optimization, Computer Methods in Applied Mechanics and Engineering199(2010) $2059-71$.

[10] W. Wall, M. Frenzel, C. Cyron, Isogeometric structural shape optimization, Computer Methods in Applied Mechanics and Engineering 197(2008) $2976-88$.

[11] Y.-D. Seo, H.-J. Kim, S.-K. Youn, Shape optimization and its extension to topological design based on isogeometric analysis, International Journal of Solids and Structures47(2010) $1618-40$.

[12] H. Gómez, V. Calo, Y. Bazilevs, T. Hughes, Isogeometric analysis of the Cahn-Hilliard phase-field model, Computer Methods in Applied Mechanics and Engineering197(2008) 4333 -52.

[13] T. Sederberg, J. Zheng, A. Bakenov, A. Nasri, T-splines and T-NURCCS, ACM Transactions on Graphics22(2003) $477-84$.

[14] T. Sederberg, D. Cardon, G. Finnigan, N. North, J. Zheng, T. Lyche, Tspline simplification and local refinement, ACM Transactions on Graphics23(2004) 276-83. 
[15] Y. Bazilevs, V. Calo, J. Cottrell, J. Evans, T. Hughes, S. Lipton, M. Scott, T. Sederberg, Isogeometric analysis using T-splines, Computer Methods in Applied Mechanics and Engineering199(2010) 229 -63.

[16] M. Dörfel, B. Jüttler, B. Simeon, Adaptive isogeometric analysis by local h-refinement with T-splines, Computer Methods in Applied Mechanics and Engineering 199(2010) $264-75$.

[17] T. Hughes, A. Reali, G. Sangalli, Efficient quadrature for NURBS-based isogeometric analysis, Computer Methods in Applied Mechanics and Engineering199(2010) $301-13$.

[18] R. Echter, M. Bischoff, Numerical efficiency, locking and unlocking of NURBS finite elements, Computer Methods in Applied Mechanics and Engineering 199(2010) $374-82$.

[19] J. Cottrell, T. Hughes, A. Reali, Studies of refinement and continuity in isogeometric structural analysis, Computer Methods in Applied Mechanics and Engineering196(2007) $4160-83$.

[20] Y. Bazilevs, L. B. da Veiga, J. Cottrell, T. Hughes, G. Sangalli, Isogeometric analysis: approximation, stability and error estimates for h-refined meshes, Mathematical Models and Methods in Applied Sciences 16(2006) $1031-90$.

[21] S. Lipton, J. Evans, Y. Bazilevs, T. Elguedj, T. Hughes, Robustness of isogeometric structural discretizations under severe mesh distortion, Computer Methods in Applied Mechanics and Engineering199(2010) 357-73.

[22] E. Cohen, T. Martin, R. Kirby, T. Lyche, R. Riesenfeld, Analysis-aware modeling: Understanding quality considerations in modeling for isogeometric analysis, Computer Methods in Applied Mechanics and Engineering 199(2010) $334-56$.

[23] J. Lu, Circular element: Isogeometric elements of smooth boundary, Computer Methods in Applied Mechanics and Engineering198(2009) $2391-402$.

[24] T. Martin, E. Cohen, R. Kirby, Volumetric parameterization and trivariate B-spline fitting using harmonic functions, Computer Aided Geometric Design26(2009) $648-64$.

[25] D. Wang, J. Xuan, An improved NURBS-based isogeometric analysis with enhanced treatment of essential boundary conditions, Computer Methods in Applied Mechanics and Engineering199(2010) 2425 -36.

[26] T. Takacs, Existence of Stiffness Matrix Integrals for Singularly Parameterized Domains in Isogeometric Analysis, Master's thesis, Johannes Kepler University Linz, Austria, 2010.

[27] S. Brenner, R. Scott, The Mathematical Theory of Finite Element Methods, Springer, 2005

[28] H. Prautzsch, W. Boehm, M. Paluszny, Bézier and B-Spline Techniques, Springer, New York, 2002.

[29] L. Piegl, W. Tiller, The NURBS book, Springer, London, 1995.

[30] J. Hoschek, D. Lasser, Fundamentals of computer aided geometric design, A. K. Peters, Ltd., Natick, 1993.

[31] M. Aigner, C. Heinrich, B. Jüttler, E. Pilgerstorfer, B. Simeon, A. V. Vuong, Swept volume parameterization for isogeometric analysis, in: Proceedings of the 13th IMA International Conference on Mathematics of Surfaces XIII, Springer, Berlin, Heidelberg, 2009, pp. 19-44. 